\title{
An Optimal Control Approach to Scheduling and Production in a Process using Decaying Catalysts
}

\author{
S. D. Adloor ${ }^{\mathrm{a}}$, T. Pons ${ }^{\mathrm{a}}$, V. S. Vassiliadis ${ }^{\mathrm{b}, *}$ \\ ${ }^{a}$ Department of Chemical Engineering and Biotechnology, University of Cambridge, \\ Philippa Fawcett Drive, Cambridge, CB3 0AS, United Kingdom \\ ${ }^{b}$ Cambridge Simulation Solutions Ltd., 8 Cody Road, Waterbeach, Cambridge, CB25 \\ 9LS, United Kingdom
}

\begin{abstract}
This article presents a novel approach to optimise scheduling and production planning to meet seasonal demand in an industrial process using decaying catalysts, based on its formulation as a multistage mixed-integer optimal control problem (MSMIOCP). Unlike existing methodologies, the MSMIOCP formulation allows to solve this problem as a standard nonlinear optimisation problem without combinatorial optimisation methods, which can be advantageous in providing reliable, robust and efficient solutions. Using this formulation, four case studies of this problem, differing in reaction or deactivation kinetics, are investigated. Two different solution implementations are used, each having their own relative advantages. The first implementation demonstrates a bang-bang behaviour for the linear scheduling controls, consistent with a theoretical analysis, but faces integration problems and does not always produce high quality solutions. The second implementation, while not demonstrating the bang-bang property, always produces high quality solutions and shows the advantages of the MSMIOCP formulation over existing methodologies.
\end{abstract}

Keywords: Optimal control problem; Bang-bang control; Mixed-integer

\footnotetext{
*Corresponding author

Email address: vsv20@cam.ac.uk (V. S. Vassiliadis)
} 
optimization; Catalyst replacement; Scheduling; Production planning

\section{Introduction}

Industrial processes that use decaying catalysts face significant negative economic setbacks. The space-time yield of the process product decreases with the time-on-stream as the catalyst deactivates, thereby causing a lower production rate and loss of revenue. Further, the process has to be shut down to load a new catalyst or regenerate the deactivated one, which can lead to a large expenditure on energy and labour.

Catalyst deactivation is inevitable and the catalyst has to be replaced in order to restore the process performance. It is necessary to minimise the costs arising from catalyst deactivation to ensure maximum profit for the process. There is a trade-off to be addressed between frequently renewing the catalyst loads to attain a high production rate and the maintenance costs and loss in production occurring from the process shut-down for catalyst changeovers. For this purpose, an efficient schedule for the replacement of the catalysts is required. In addition, an optimal production plan is needed, that details the operating conditions of the process while taking into account the catalyst deactivation and the process economics.

Studies to minimise the negative effects of catalyst deactivation have previously been carried out at the reactor or pilot plant level. Szépe and Levenspiel (1968) were the first to identify the optimal temperature policy to maximise the conversion of the reactant in a batch reactor containing a deactivating catalyst. They considered the reaction kinetics to be separable from the catalyst activity and a deactivation rate law that was independent of the concentration of the species involved. They demonstrated that if the deactivation kinetics is more sensitive to temperature than the main reaction, then it is optimal to continuously increase the temperature of operation so as to 
keep the effective reaction rate constant unchanged throughout the reaction cycle. However, if the deactivation kinetics is less sensitive to temperature than the main reaction, the optimal temperature policy is to operate at the maximum temperature limit. Further, they applied this condition to stirred flow reactors and established a policy of maintaining constant reactant exit conversion, by varying either the flow-rate or the temperature.

Other studies (Chou et al., 1967; Crowe, 1970; Crowe and Lee, 1971) have similarly derived maintaining a constant reactant exit conversion as the optimal policy for tubular reactors using decaying catalysts. Lee and Crowe (1970) considered, for batch reactors, a more complicated form of deactivation kinetics, which was dependent on species' concentration, and concluded that a constant effective rate coefficient was no longer an optimal policy. Crowe (1976) however, reported that for continuous stirred and plug flow reactors, even when concentration dependent deactivation is involved, constant exit conversion remains the optimal policy under certain conditions. Further works (Krishnaswamy and Kittrell, 1979; Ho, 1984; Pacheco and Petersen, 1986; Sapre, 1997) have been published, which obtain and analyse a relation between the time-on-stream and the temperature of operation, while assuming constant exit conversion as the optimal operating policy, for flow reactors containing deactivating catalysts.

All of the aforementioned publications have focused on identifying the optimal operating policy to maximise the conversion of the reactant, until when the temperature of operation reaches its upper limit or when the catalyst has to be discarded or replaced. On an industrial scale, however, such strategies may not constitute the optimal policy as other aspects have to be taken into consideration such as the seasonal demand figures and the storage costs. For instance, maintaining a constant production rate may result in a high inventory level during low demand seasons. This could also cause the 
catalyst to be used up very fast. Hence, it is desired to plan production, such that the production rate is not too high during low demand seasons while also maintaining an inventory level sufficient to meet the demand during times of plant shutdowns for catalyst changeovers. The scheduling of catalyst replacements along with the plant operating conditions (temperature and flow rate) should be organised such that the production level meets seasonal demand in an efficient manner and makes maximum use of the catalyst life.

Most available literature that address the scheduling of catalyst changeovers and production planning on an industrial scale are based on Mixed-Integer Nonlinear Programming (MINLP) methodologies. Lang et al. (2000) have developed an optimal catalyst management policy for an Oxo process. But this work does not consider planning production to meet time-varying demand. Houze et al. (2003) formulated a model using the big-M formulation to schedule catalyst changeovers and plan production to meet seasonal demand for 2-year and 4-year horizons. Bizet et al. (2005) modified the model in Houze et al. (2003) by using convex hull formulations instead of the big-M formulations wherever possible, which enabled solutions for longer time horizons of 74-months and 9-years. Further, they claim, without rigorous proof, to overcome the non-convexities of that model to obtain global optimality by using two different approaches: a partitioning search strategy and the Generalized Benders Decomposition (Geoffrion, 1972).

In what could be applicable to the problem discussed here, recent publications have showcased advancements in MINLP techniques which, they say, can facilitate convergence in the optimisation of production planning and scheduling for large scale problems. Su et al. (2015) have presented strategies such as multiple-generation cuts, hybrid methods and partial surrogate cuts for improving the efficiencies of the Outer Approximation and Generalized Benders Decomposition methods and Su et al. (2016) have applied 
one of these techniques in a cracking production process. Other developments such as cutting plane methods (Eronen et al., 2015) and supporting hyperplane techniques (Westerlund et al., 2018) claim to produce easier convergence in nonsmooth, generalised convex formulations and demonstrate applicability to production and scheduling problems. Other methodologies for facilitating solutions in MINLP formulations of planning and scheduling problems include Lagrangian decomposition techniques (e.g. Mouret et al. (2011), Wang et al. (2016)), bi-level decomposition methods (e.g. Li and Ierapetritou (2009), Shi et al. (2015), Lin and Du (2018)) and rolling horizon methods (e.g. Al-Ameri et al. (2008), Li and Ierapetritou (2010)).

The use of MINLP approaches, as done in the aforementioned publications, requires all differential equations present to be discretised and imposed as equality constraints under a steady state assumption. This "infeasible path approach" to solving the differential equations causes the problem to have a very large number of variables and nonlinear constraints, especially when long time horizons are considered. This could lead to convergence difficulties. Further, the steady state assumption prevents an accurate description of the process dynamics within the time period of discretisation. In addition, an increase in the number of catalysts involved would accentuate these problems due to an exponential increase in the number of scenarios. Most publications also do not reveal their kinetic model or parameters, due to confidentiality clauses, and this prevents the reproduction and validation of their results.

The preceding discussion indicates that there is a need for a robust, reliable and efficient solution methodology to the catalyst replacement scheduling optimisation problem. The methodology should be able to predict (i) the number of catalyst loads to use and an efficient schedule for the catalyst changeovers (ii) the optimal plant operating conditions of flow rate and temperature at regular intervals and (iii) the production and inventory levels to 
meet seasonal demand effectively.

Such predictions should be possible even for long time horizons and complex reaction kinetics. This is the focus of this article. A novel solution methodology is proposed based on the realisation that the catalyst replacement scheduling problem is in actuality a Multistage Mixed-Integer Optimal Control Problem (MSMIOCP). Such a formulation can provide the advantages of robustness, reliability and efficiency over existing MINLP techniques by using state-of-the-art integrators and negating the use of combinatorial optimisation methods. In fact, this methodology can be applied to any decaying performance maintenance scheduling optimisation problem.

The rest of the paper is organised as follows. In Section 2 the multistage mixed-integer optimal control formulation of this problem is developed. In Section 3 this formulation is applied to different case studies of an industrial process, and the solution implementation methodologies and results obtained are discussed. Section 4 contains the conclusions of this work, which also details the advantages of the proposed approach over previous methodologies. For the interested readers, a theoretical analysis of the MSMIOCP formulation is done in Appendix A and Appendix B contains a set of tables which would aid in reproducing the results obtained in this work.

\section{An optimal control approach to the catalyst replacement schedul- ing and production planning problem}

In this section, the catalyst replacement scheduling problem is developed as an MSMIOCP, characterised by a set of decision and state variables. The whole time horizon is divided into stages, with each stage being described by a process model constituted by the appropriate Differential Algebraic Equations (DAEs), constraints, initial conditions and junction conditions that link any two consecutive stages. For each stage, a decision has to be made on 
whether the catalyst should be in operation or a shut down occurs. Further, the plant operating conditions and the amount of product sales should also be decided at each stage. These decision variables, when chosen optimally, result in the maximum profit or the minimum costs for the process.

A control parametrisation approach is adopted wherein the decision variables are discretised over the whole time horizon at the times corresponding to each stage while the state variables are retained in their continuous form, to be solved by an integrator. The DAEs are solved to a high accuracy in the right sequential order and hence, this solution methodology is called a "feasible path approach" (Vassiliadis, 1993; Vassiliadis et al., 1994a,b).

The catalyst changeover decisions appear linearly in the system equations and so are expected to take values at either bound, thus exhibiting binary nature and lending what is called a bang-bang nature to the solution. However, the other controls may not appear linearly in the system equations and so may appear in a continuous form without exhibiting such a bang-bang behaviour. The key feature is that the bang-bang behaviour enables the relaxation of the integer restrictions of the MSMIOCP and its solution as a standard Nonlinear Programming (NLP) problem, by avoiding the need for combinatorial optimisation methods to schedule catalyst changeovers. The formulation as an MSMIOCP follows next.

The basic formulation for an OCP is shown in equations (1a) - (1d). The performance index consists of a point index $\phi$ and a continuous index $L$. This performance index is minimised by the selection of controls $w(t)$ subject to differential and algebraic equations, $h$ and $g$, involving differential and algebraic state variables, $x(t)$ and $z(t)$, respectively. The controls $w(t)$ can include linear controls $u(t)$ that are binary in nature as well as nonlinear controls $v(t)$, which can take continuous values. Equations (1b) - (1d) 
describe an index-1 DAE system, given initial condition $x_{0}$ and fixed initial and final times, $t_{0}$ and $t_{F}$, respectively.

$$
\min _{w(t)} W=\phi\left(x\left(t_{F}\right)\right)+\int_{t_{0}}^{t_{F}} L(x(t), z(t), w(t), t) \mathrm{d} t
$$

subject to

$$
\dot{x}(t)=h(x(t), z(t), w(t), t), \quad x\left(t_{0}\right)=x_{0}
$$

$$
\begin{gathered}
g(x(t), z(t), w(t), t)=0 \\
w(t)=\left[[u(t)]^{T},[v(t)]^{T}\right]^{T}, \quad u(t) \in \mathcal{U}, \quad \mathcal{U} \in\{0,1\}, \quad \forall t \in\left[t_{0}, t_{F}\right]
\end{gathered}
$$

A multistage form is obtained by discretisation of the scheduling horizon into time periods (which can be of arbitrary lengths), where the control profiles are allowed to be discontinuous at a finite number of points, $t_{p}$, termed junctions. A general form of junction conditions between any two consecutive periods, $p$ and $p+1$, as given by Vassiliadis (1993), is used here, as per equation (2):

$$
\begin{gathered}
J\left(\dot{x}_{p+1}\left(t_{p}^{+}\right), x_{p+1}\left(t_{p}^{+}\right), z_{p+1}\left(t_{p}^{+}\right), w_{p+1}\left(t_{p}^{+}\right)\right. \\
\left.\dot{x}_{p}\left(t_{p}^{-}\right), x_{p}\left(t_{p}^{-}\right), z_{p}\left(t_{p}^{-}\right), w_{p}\left(t_{p}^{-}\right), t_{p}\right)=0 \\
\forall p=1,2, \ldots N P-1
\end{gathered}
$$

The basic form of the multistage OCP over time periods, $p=1,2, \ldots N P$, $t \in\left[t_{p-1}, t_{p}\right]$, with $t_{N P}=t_{F}$ is shown in equations $(3 \mathrm{a})-(3 \mathrm{~g})$. The performance index and differential algebraic equations are presented in a form that explicitly shows the linearity of the control $u^{(p)}$, for stage $p$. An illustration of the MSMIOCP formulation is shown in Figure 1. 


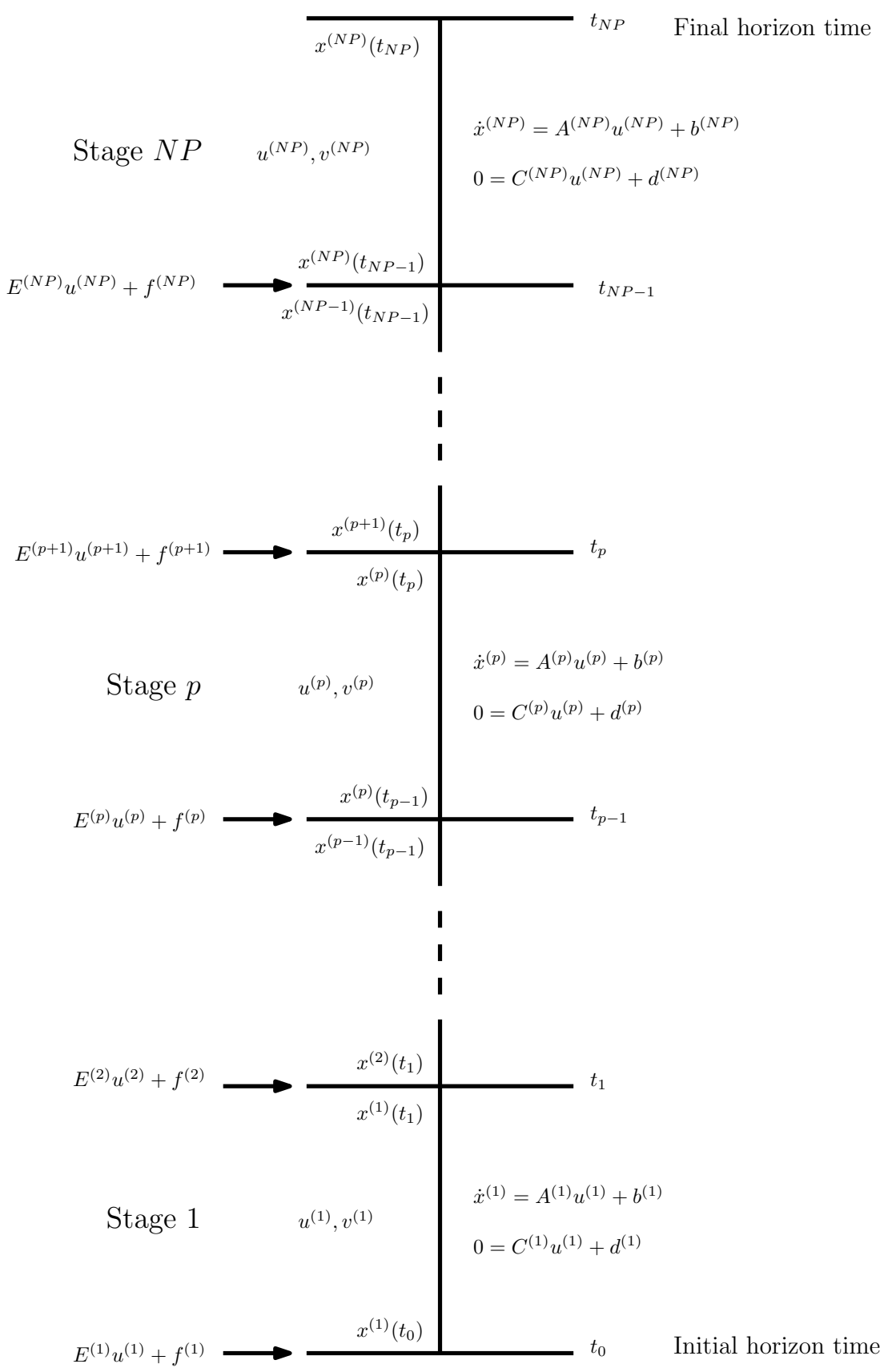

Figure 1: An illustration of the MSMIOCP formulation 


$$
\begin{aligned}
\min _{u, v} W & =\sum_{p=1}^{N P}\left\{\phi^{(p)}\left(x^{(p)}\left(t_{p}\right), z^{(p)}\left(t_{p}\right), w^{(p)}, t_{p}\right)+\int_{t_{p-1}}^{t_{p}} L^{(p)}\left(x^{(p)}(t), z^{(p)}(t), w^{(p)}, t\right) \mathrm{d} t\right\} \\
& =\sum_{p=1}^{N P}\left\{\left[\phi_{1}^{(p)}\left(x^{(p)}\left(t_{p}\right), z^{(p)}\left(t_{p}\right), v^{(p)}, t_{p}\right)\right]^{T} u^{(p)}+\phi_{2}^{(p)}\left(x^{(p)}\left(t_{p}\right), z^{(p)}\left(t_{p}\right), v^{(p)}, t_{p}\right)\right. \\
& \left.+\int_{t_{p-1}}^{t_{p}}\left[\left[L_{1}^{(p)}\left(x^{(p)}(t), z^{(p)}(t), v^{(p)}, t\right)\right]^{T} u^{(p)}+L_{2}^{(p)}\left(x^{(p)}(t), z^{(p)}(t), v^{(p)}, t\right)\right] \mathrm{d} t\right\}
\end{aligned}
$$

199

subject to

$$
\dot{x}^{(p)}(t)=A^{(p)}\left(x^{(p)}(t), z^{(p)}(t), v^{(p)}, t\right) u^{(p)}+b^{(p)}\left(x^{(p)}(t), z^{(p)}(t), v^{(p)}, t\right)
$$

$$
0=C^{(p)}\left(x^{(p)}(t), z^{(p)}(t), v^{(p)}, t\right) u^{(p)}+d^{(p)}\left(x^{(p)}(t), z^{(p)}(t), v^{(p)}, t\right)
$$

$$
x^{(1)}\left(t_{0}\right)=E^{(1)}\left(v^{(1)}\right) u^{(1)}+f^{(1)}\left(v^{(1)}\right)
$$

$$
\begin{aligned}
x^{(p)}\left(t_{p-1}\right) & =E^{(p)}\left(x^{(p-1)}\left(t_{p-1}\right), z^{(p-1)}\left(t_{p-1}\right), v^{(p)}\right) u^{(p)} \\
& +f^{(p)}\left(x^{(p-1)}\left(t_{p-1}\right), z^{(p-1)}\left(t_{p-1}\right), v^{(p)}\right) \\
p & =2,3, \ldots, N P
\end{aligned}
$$

$$
u(t) \in \mathcal{U}, \quad \mathcal{U} \in\{0,1\}
$$

In equation (3a), the point performance index is represented as functions of $\phi_{1}$ and $\phi_{2}$, where $\phi_{1}$ is the coefficient of the linear control and both terms are themselves independent of the linear controls. $L_{1}$ and $L_{2}, A$ and $b, C$ and $d, E$ and $f$ are the analogous terms for the continuous performance index, the differential equations, the algebraic equations and the junction conditions, respectively. 
The stage switching times, $t_{p}$ are considered to be constant in this derivation. The controls $u^{(p)}$ and $v^{(p)}$ are considered to be piecewise constant. The control $u^{(p)}$ is binary in nature and indicates whether a catalyst is in operation $\left(u^{(p)}=1\right)$ or is being replaced $\left(u^{(p)}=0\right)$. The control $v^{(p)}$ is continuous and represents the operating conditions of the process. The collective vector of controls, $u$ and $v$, over all stages is:

$$
u=\left[u^{(1)}, u^{(2)}, \ldots, u^{(N P)}\right]^{T}
$$

$$
v=\left[v^{(1)}, v^{(2)}, \ldots, v^{(N P)}\right]^{T}
$$

A theoretical analysis that applies the Pontryagin Minimum (Maximum) principle (Pontryagin et al., 1962) is done in Appendix A, similar to that done by Al Ismaili et al. (2018) and Adloor et al. (2018). The difference here is that the controls are distinguished as occurring linearly or nonlinearly, whereas those works considered only linear controls. As can be seen in equation (A.14a), the affine controls $u$, when differentiated, do not participate in a bilinear or product form with the nonlinear controls $v$. Hence, despite the interaction between the linear and nonlinear controls in the system equations, the Hamiltonian gradient with respect to $u^{(p)}$ is independent of that linear control. This expression can be termed a "switching function" in the sense that it can cause the value of $u^{(p)}$ to switch in order to minimise the Hamiltonian. Some notable points:

1. If the switching function is positive or negative, the Hamiltonian is minimised when the control $u^{(p)}$ is at its lower or upper bound, respectively. This phenomenon of an optimal control action occurring at either bound of the feasible region is called "bang-bang" control (Bryson and Ho, 1975).

2. There may be some stages where the switching function becomes zero, thus resulting in the Hamiltonian gradient at that stage to become 
insensitive to variations in $u$. In such cases, a bang-bang behaviour may not be observed and the stage is called a singular arc.

Thus, the condition $(3 \mathrm{~g})$ for the MSMIOCP can be relaxed to a form:

$$
u(t) \in \mathcal{U}^{\prime}, \quad \mathcal{U}^{\prime} \in[0,1]^{\operatorname{dim}[u(t)]}
$$

The optimal control for the relaxed MSMIOCP with respect to the linear controls $u$, can be expected to exhibit a bang-bang behaviour with potential singular arcs.

However, as can be seen in equation (A.13a), the Hamiltonian gradient with respect to the control $v^{(p)}$, which appeared nonlinearly in the system equations, is not independent of this control. Hence, the controls $v$ are not expected to exhibit a bang-bang behaviour.

The phenomenon of pure bang-bang controls have previously been demonstrated in minimum time problems for linear (Bellman et al., 1956) and bilinear systems (Mohler, 1973), in the optimal control of a batch reactor (Blakemore and Aris, 1962), optimal thermal control (Belghith et al., 1986) and in the optimal drug administration for cancer chemotherapy Ledzewicz and Schättler (2002). Zandvliet et al. (2007), however, in an application to reservoir flooding problems, have shown that when controls come linearly in relation to the continuous state variables, if the only constraints on the controls are upper and lower bounds, then bang-bang solutions can occur in combination with singular arcs. Thus, the predictions of the Pontryagin analysis carried out here is consistent with those of Zandvliet et al. (2007).

Sager (2009) has presented a methodology to handle nonlinear dynamic systems involving discrete and continuous controls. Techniques are presented to reformulate the problem to avoid nonlinearities and enforce discrete con- 
trols via auxiliary binary controls that occur linearly in the system dynamics and exhibit a bang-bang behaviour. Heuristics, e.g. rounding or sum up rounding strategies or algorithms such as Branch and Bound are used to ensure integer solutions when singular arcs appear. This methodology has been used in a variety of applications (Sager et al., 2009; Kirches et al., 2010; Sager, 2005). In this article, however, there is no need for any such reformulation because the discrete controls already occur linearly in the system equations. It is worth mentioning, however, that the Pontryagin analysis' predictions of bang-bang behaviour for the linear controls, even when in combination with other continuous controls, are consistent with those of Sager (2009).

The formulation of the catalyst replacement scheduling problem as a relaxed MSMIOCP offers a number of advantages over previous methodologies:

1. The feasible path approach employs state-of-the-art integrators to solve the differential equations, thereby giving highly accurate solutions. The dynamic nature of the process is addressed in exactness, unlike in the MINLP formulations which discretise the differential equations under a steady state assumption.

2. The infeasible path approach adopted by the existing methodologies, which imposes the discretised differential equations as equality constraints, causes the problem to have a very large number of nonlinear constraint equations. This leads to convergence difficulties. In contrast, in the feasible path approach, the differential equations are solved by an integrator without being considered as constraints in the optimisation phase. The resulting problem is of a much smaller size and convergence can be obtained even from random start points. Thus, the proposed approach is more robust compared to other methodologies.

3. The bang-bang behaviour avoids the need for combinatorial optimisation methods to schedule the catalyst changeovers. Thus, this is more 
efficient than other approaches as no computational effort is spent in deciding when to schedule catalyst changeovers.

Thus, the formulation as a relaxed MSMIOCP has great potential for offering a reliable, robust and efficient solution to the catalyst replacement scheduling problem. Of course, global optimality of the solution cannot be guaranteed by this methodology but even the MINLP formulations presented previously suffer from this shortcoming.

The analysis as a relaxed MSMIOCP is general to any maintenance scheduling problem formulation that has the same model structure and hence it opens up the way to address other challenging problems. Al Ismaili et al. (2018) have demonstrated this for a heat exchanger network cleaning scheduling problem, where the controls are cleaning actions that appear linearly in the system dynamics and so, exhibit a bang-bang behaviour. In the following section, this formulation is applied to different case studies of a catalyst replacement scheduling optimisation problem.

\section{Case Studies}

In this section, the relaxed MSMIOCP formulation of the catalyst replacement scheduling problem is applied in case studies to maximise the profit of an industrial process that uses a decaying catalyst to produce the desired product. The essential elements of the problem formulation are discussed first before presenting the results obtained.

\subsection{Problem formulation}

In the problem addressed, the following assumptions apply:

1. The industrial process operates over a fixed time horizon, in the order of years. Each year is constituted by 12 months and there are a total of $N M$ months, wherein each month is constituted by 4 weeks. 
2. The industrial process functions according to a certain process model and is subject to operating constraints.

3. The reactor containing the deactivating catalyst is a Continuous Stirred Tank Reactor (CSTR) that is of known and fixed volume.

4. The catalyst performance decays with time and has to be replaced before it crosses a certain maximum age. Various forms of catalyst deactivation kinetics will be investigated in the different case studies.

5. The catalyst deactivation rate constant is taken to be independent of the temperature of operation.

6. There is a maximum number of catalyst loads that can be used over the given time horizon.

7. All available catalysts exhibit identical functioning and performance.

8. The time required to shut down the process, replace the catalyst and restart the process is taken to be one month, during which time no production occurs.

9. The main reaction is assumed to be of the form:

$$
R \rightarrow Q
$$

where $R$ is the reactant and $Q$ is the desired product. The different case studies will examine first and second order kinetics with respect to the reactant's concentration. Further, in each case study, the reaction rate will be considered separable from the catalyst activity.

10. The reaction rate constant is taken to exhibit an Arrhenius form of temperature dependence.

11. The feed inlet concentration is taken to be known and constant. 
12. The flow rate of raw material to the reactor has to be specified on a weekly basis.

13. The flow rate of raw material to the reactor has an upper limit during catalyst operation and is stopped when the catalyst is being replaced.

14. The temperature of the reactor has to be specified on a weekly basis.

15. The temperature of the reactor can be operated only within fixed bounds during catalyst operation and is set to its lower bound during catalyst replacement.

16. The product is produced and stored continuously as inventory.

17. The product produced is sold on a weekly basis.

18. The seasonal demand figures for the product are given.

19. The sales for each week is less than or equal to the customer demand for the product in that week.

20. There is a penalty corresponding to the unmet demand in each period.

21. The costs involved in the process are known and are subject to a known value of annual inflation. These include the sales price of the product, the cost of inventory, the cost of flow and raw material, the cost of catalyst changeover and the penalty for unmet demand.

Given the above assumptions, the optimisation model must determine the following values, which constitute the controls of the MSMIOCP:

(i) The catalyst changeover decision variable, $y(i)$, for each month, $i$, which determines whether a catalyst is in operation $(y(i)=1)$ or being replaced $(y(i)=0)$ during that month.

(ii) The feed flow rate to the reactor, $f f r(i, j)$, during each week, $j$, of each month, $i$. 
(iii) The temperature of operation of the reactor, $T(i, j)$, during each week, $j$, of each month, $i$.

(iv) The amount of product sold, sales $(i, j)$, at the end of each week, $j$, of each month, $i$.

In the above list, $j \in\{1,2,3,4\}$ and $i \in\{1,2, \ldots, N M\}$. The catalyst changeover decisions correspond to the binary controls $u$ in equation (4a) while the other decision variables correspond to continuous controls $v$ in equation (4b).

The state variables that characterise the MSMIOCP formulation of this industrial process include (i) the catalyst age, cat_age (ii) the catalyst activity, cat_act (iii) the concentration of the reactant at the exit of the reactor, $c R$ (iv) the inventory level, inl and (v) the cumulative inventory costs, cum_inc. These state variables are determined by the decision variables' values at any time using a set of Ordinary Differential Equations (ODEs) which constitute the process model. In the following, process models to describe different case studies of the industrial process are formulated. These ODEs apply for week $j \in\{1,2,3,4\}$ of month $i \in\{1,2, \ldots, N M\}$ of the process and are of the form of equation (3b). Unless specified, a particular model equation applies to all case studies:

1. The catalyst age varies linearly with time when the catalyst is in operation $(y(i)=1)$ but does not increase at times of catalyst replacement $(y(i)=0)$. Hence, the differential equation describing the catalyst age at all times is given by:

$$
\frac{d(\text { cat_age })}{d t}=y(i)
$$

2. The catalyst activity decays according to a deactivation rate law during times of catalyst operation $(y(i)=1)$ but experiences no change during 
times of catalyst replacement $(y(i)=0)$, when there is no production occurring. Thus, the differential equation for the catalyst activity takes the form:

$$
\frac{d\left(c a t_{-} a c t\right)}{d t}=y(i) \times r D
$$

where $r D$ is the rate of catalyst deactivation. Different models of catalyst deactivation kinetics are considered as separate case studies:

Case Study A: Composition independent catalyst deactivation

$$
r D=-K_{d} \times c a t_{-} a c t
$$

Case Study B: Reactant concentration dependent catalyst deactivation

$$
r D=-K_{d} \times c a t_{-} a c t \times c R
$$

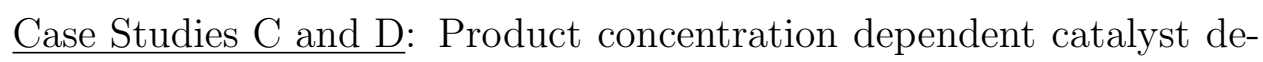
activation

$$
r D=-K_{d} \times c a t_{-} a c t \times(C R 0-c R)
$$

where $K_{d}$ is the deactivation rate constant and $C R 0$ is the reactant entry concentration.

3. The reactor is assumed to be completely stirred and so the reactant exit concentration $(c R)$ is obtained from the generic mass balance equation of a CSTR during times of catalyst operation $(y(i)=1)$. However, during catalyst replacement $(y(i)=0)$, no reaction occurs and the reactor is assumed to be filled with fresh, unreacted reactant at the entry concentration $(C R 0)$, to be used by the new catalyst after replacement. The differential equation that accounts for both scenarios is given by: 


$$
\frac{d(V R \times c R)}{d t}=(f f r(i, j) \times(C R 0-c R))-(y(i) \times V R \times r R)
$$

where $V R$ is the volume of the reactor and $r R$ is the rate of reaction (6). The case studies consider different forms of $r R$ :

Case Studies A, B and C: First order kinetics for reaction (6)

$$
r R=K_{1} \times \text { cat_act } \times c R
$$

Case Study D: Second order kinetics for reaction (6)

$$
r R=K_{1} \times c a t_{-} a c t \times c R^{2}
$$

where $K_{1}$ is the rate constant. For all case studies, $K_{1}$ is assumed to exhibit an Arrhenius form of temperature dependence, of the form:

$$
K_{1}=A_{R} \times \exp \left(-\frac{E_{a c t}}{R_{g} \times T(i, j)}\right)
$$

where $A_{R}$ is the pre-exponential factor, $E_{a c t}$ is the activation energy for the reaction and $R_{g}$ is the universal gas constant.

4. It is assumed that whatever product is produced is stored as inventory before being sold at the end of the week. During catalyst operation $(y(i)=1)$, the increase in inventory level at any time depends on the rate of production $(=V R \times r R)$ of the product chemical, but during catalyst replacement $(y(i)=0)$, there is no increase in inventory level. Hence, the differential equation that provides a description of the inventory level $(\mathrm{inl})$ for both scenarios is given by:

$$
\frac{d(i n l)}{d t}=y(i) \times(V R \times r R)
$$


where the expression for $r R$ depends on the case study.

5. Finally, the increase in the cumulative inventory cost (cum_inc) at any time depends on the inventory level at that time and the Inventory Cost Factor (icf) (adjusted for inflation), which stipulates the cost per unit product per unit time:

$$
\frac{d\left(c u m_{-} i n c\right)}{d t}=i n l \times i c f
$$

The $i c f$ at any time is given by the following equation:

$$
i c f=\text { base_icf } \times(1+\text { inflation })^{\lfloor i / 12\rfloor}
$$

where base_icf is the inventory cost factor before inflation, inflation is the annual inflation rate and $\lfloor\cdot\rfloor$ is the greatest integer function.

For each case study, the process model is solved repeatedly over a weekly time span, which corresponds to one stage of the MSMIOCP. In order to solve these ODEs, for each stage, suitable initial conditions have to be provided. The initial conditions for week 1 of month 1 are assumed to be known and are of the form of equation (3e). The initial conditions for the other stages are obtained using junction conditions between two successive stages of the process, of the form of equation (3f).

The initial conditions corresponding to week 1 of month 1 , represented as init_var $(1,1)$ for variable var, are as follows:

1. The initial catalyst age is that of a fresh catalyst, which is zero:

$$
\text { init_cat_age }(1,1)=0
$$


2. The initial catalyst activity is that of a fresh catalyst (start_cat_act):

$$
\text { init_cat_act }(1,1)=\text { start_cat_act }
$$

3. At the start of the process, the reactor is filled with the reactant $R$ at its entry concentration $C R 0$. Hence, the initial exit concentration is given by:

$$
\text { init_cR }_{-}(1,1)=C R 0
$$

4. There is no inventory at the beginning of the process, and so:

$$
\text { init_inl }(1,1)=0
$$

5. There is no inventory at the start of the process and so the initial cumulative inventory cost is given by:

$$
\text { init_cum_inc }(1,1)=0
$$

The junction conditions are described next. These junction conditions differ depending on whether the catalyst is in operation $(y(i)=1)$ or is being replaced $(y(i)=0)$ during that month. In the following text, the expressions init_var $(i, j)$ and end_var $(i, j)$ indicate the initial and end conditions, respectively for the variable var, for week $j$ of month $i$ :

1. During months of catalyst operation $(y(i)=1)$, the initial catalyst age for a week corresponds to the catalyst age at the end of the previous week. But during months of catalyst replacement $(y(i)=0)$, the catalyst age has to be set to zero, the age of a new catalyst. The junction conditions that describe both scenarios is given by: 


$$
\begin{gathered}
\text { init_cat_age }(i, j+1)=\text { end_cat_age }(i, j) \\
\forall j=1,2,3 \quad \forall i=1,2, \ldots, N M
\end{gathered}
$$

459

$$
\begin{aligned}
\text { init_cat_age }(i, 1) & =\left[y(i) \times e n d_{-} \text {cat_age }(i-1,4)\right] \\
\forall i & =2,3, \ldots, N M
\end{aligned}
$$

460

2. During months of catalyst operation $(y(i)=1)$, the initial catalyst activity for the week corresponds to the catalyst activity at the end of the previous week. However, during months of catalyst replacement $(y(i)=0)$, the catalyst activity has to be reset to the activity corresponding to that of a fresh catalyst, which remains the same throughout the duration of month $i$. The junction conditions that describe both scenarios is given by:

$$
\begin{gathered}
\text { init_cat_act }(i, j+1)=\text { end_cat_act }(i, j) \\
\forall j=1,2,3 \quad \forall i=1,2, \ldots, N M
\end{gathered}
$$

$$
\begin{gathered}
\text { init_cat_act }(i, 1)=\left[y(i) \times e n d_{-} c a t_{-} a c t(i-1,4)\right]+[(1-y(i)) \times \text { start_cat_act }] \\
\forall i=2,3, \ldots, N M
\end{gathered}
$$

3. During months of catalyst operation $(y(i)=1)$, the exit concentration for the beginning of a week corresponds to the exit concentration at the end of the previous week. And during months of catalyst replacement $(y(i)=0)$, the reactor is filled with reactant at entry concentration $C R 0$, ready to be used by the fresh catalyst at the beginning of the next month. So, the junction conditions take the form:

$$
\begin{aligned}
& \text { init_cR }(i, j+1)=\text { end_cR }(i, j) \\
& \forall j=1,2,3 \quad \forall i=1,2, \ldots, N M
\end{aligned}
$$




$$
\begin{gathered}
\text { init_cR(i,1)=[y(i) } \left.\times e^{e n d} d_{-} c R(i-1,4)\right]+[(1-y(i)) \times C R 0] \\
\forall i=2,3, \ldots, N M
\end{gathered}
$$

4. At the end of a week, an amount, $\operatorname{sales}(i, j)$ of the stored product is sold. Thus, the initial inventory level for the week corresponds to the inventory present after the sales at the end of the previous week. The following junction conditions apply during months of catalyst operation as well as catalyst replacement, as the sales do not cease at any time:

$$
\begin{gathered}
\text { init_inl }(i, j+1)=\text { end_inl }(i, j)-\operatorname{sales}(i, j) \\
\forall j=1,2,3 \quad \forall i=1,2, \ldots, N M
\end{gathered}
$$

$$
\begin{aligned}
& \text { init_inl }(i, 1)=\text { end_inl }(i-1,4)-\operatorname{sales}(i-1,4) \\
& \forall i=2,3, \ldots, N M
\end{aligned}
$$

5. The inventory cost accumulated until the beginning of a week is equal to the value of the inventory cost accumulated until the end of the previous week and the following junction conditions apply regardless of whether the catalyst is being used or replaced:

$$
\begin{gathered}
\text { init_cum_inc }(i, j+1)=\text { end_cum_inc }(i, j) \\
\forall j=1,2,3 \quad \forall i=1,2, \ldots, N M
\end{gathered}
$$

$$
\begin{gathered}
\text { init_cum_inc }(i, 1)=\text { end_cum_inc }(i-1,4) \\
\forall i=2,3, \ldots, N M
\end{gathered}
$$

The initial conditions $(20)$ - (23) and junction conditions (25) - (28) enable a solution for the ODEs for all stages, and thereby obtain the values of the state variables for the entire time horizon. These are then used to 
compute the values of some of the constraints and the objective function of the problem, whose formulations are described next.

The constraints that apply to this industrial process for week $j \in\{1,2,3,4\}$ of month $i \in\{1,2, \ldots, N M\}$ are as follows:

1. In the context of the formulation as a relaxed MSMIOCP, the catalyst changeover decision variables $y(i)$, for a month $i$, are considered continuous variables that vary between 0 and 1 (but are expected to take only 0 or 1 values due to the bang-bang nature of the formulation), and so the following bounds are imposed:

$$
0 \leq y(i) \leq 1
$$

2. The flow rate of raw material to the reactor has an upper limit $(F U p)$ at which it can operate. Hence, the following bounds are set on the feed flow rate for each week:

$$
0 \leq f f r(i, j) \leq F U p
$$

3. The sales in each week are assumed to be less than or equal to the demand for the product in that week $(\operatorname{demand}(i, j))$. Hence, the following bounds on the sales at the end of each week are imposed:

$$
0 \leq \operatorname{sales}(i, j) \leq \operatorname{demand}(i, j)
$$

4. The temperature of the reactor operates between known, fixed lower and upper bounds, TLo and TUp, respectively. Hence, the following bounds are set on the weekly temperature of operation of the reactor:

$$
T L o \leq T(i, j) \leq T U p
$$


5. During times of catalyst replacement, the process is shut down and so the flow of raw material to the reactor stops. The following constraint ensures that the weekly feed flow rate remains below the upper bound during times of catalyst operation $(y(i)=1)$ and drops to zero when there is catalyst replacement $(y(i)=0)$.

$$
f f r(i, j)-[F U p \times y(i)] \leq 0
$$

6. When the process is shut down for catalyst replacement, the temperature of the reactor is required to drop to its lower bound. This condition is imposed using the following constraint which ensures that the temperature for the week remains between its bounds during times of catalyst operation $(y(i)=1)$ and drops to the lower bound when there is catalyst replacement $(y(i)=0)$ :

$$
T L o \leq T(i, j) \leq[(T U p-T L o) \times y(i)]+T L o
$$

7. There is only a certain number of catalysts available to be used by the process. The limit on the maximum number of catalyst changeovers $(n)$ allowed is imposed using the following constraint:

$$
\sum_{i=1}^{N M} y(i) \geq N M-n
$$

8. The catalyst undergoes deactivation over time and has to be replaced before it crosses a certain maximum age (max_cat_age). As the the decision on whether to replace a catalyst or not is made on a monthly basis, it is sufficient to ensure that the catalyst age does not cross this limit at the end of each month $i$ :

$$
\text { end_cat_age }(i, 4) \leq \text { max_cat_age }
$$


9. In order to ensure that more product than available is not sold, the inventory level at the end of each week should be greater than the sales for the week. This is imposed using the following constraint:

$$
\operatorname{end} i \operatorname{inl}(i, j)-\operatorname{sales}(i, j) \geq 0
$$

The objective function that represents the net costs of the industrial process, is of the form of equation (3a) and comprises the following elements:

1. The Gross Revenue from Sales $(G R S)$

This term represents the revenue for the process from the net sales of the product chemical over the whole time horizon:

$$
G R S=\sum_{i=1}^{N M} \sum_{j=1}^{4} p s p(i, j) \times \operatorname{sales}(i, j)
$$

where $\operatorname{psp}(i, j)$ is the sales price per unit product for week $j$ of month $i$, adjusted for inflation at that time:

$$
p s p(i, j)=\text { base_psp } \times(1+\text { inflation })^{\lfloor i / 12\rfloor}
$$

where base_psp is the unit product sales price before inflation.

2. The Total Inventory Costs (TIC)

This term represents the net storage costs for the product over the whole time horizon and is obtained from the solution of the ODEs for the state variable cum_inc at the end of the final week of the process:

$$
T I C=\text { end_cum_inc }(N M, 4)
$$

\section{The Total Costs of Catalyst Changeovers $(T C C C)$}

The total expenditure for the catalyst changeover operations is: 


$$
T C C C=\sum_{i=1}^{N M} \operatorname{crc}(i) \times(1-y(i))
$$

where $\operatorname{crc}(i)$ is the cost of the catalyst replacement operation for month $i$, adjusted for inflation at that time:

$$
\operatorname{crc}(i)=\text { base_crc } \times(1+\text { inflation })^{\lfloor i / 12\rfloor}
$$

where base_crc is the cost of a catalyst changeover operation before inflation. It is highlighted that the terms within the summation remain non-zero only during the times of catalyst replacement $(y(i)=0)$ and only these terms contribute to the total costs.

4. The Net Penalty for Unmet Demand (NPUD) The unmet demand in each week (unmet_demand $(i, j)$ ) is the quantity of product by which the sales falls short of the demand in that week:

$$
\begin{gathered}
\text { unmet_demand }(i, j)=\text { demand }(i, j)-\operatorname{sales}(i, j) \\
\forall j=1,2,3,4 \quad \forall i=1,2, \ldots, N M
\end{gathered}
$$

There is a penalty associated with this unmet demand and the net penalty costs over the entire time horizon is given by:

$$
N P U D=\sum_{i=1}^{N M} \sum_{j=1}^{4} \operatorname{pen}(i, j) \times \text { unmet_demand }(i, j)
$$

where $\operatorname{pen}(i, j)$ is the penalty per unit product for week $j$ of month $i$, adjusted for inflation at that time:

$$
\operatorname{pen}(i, j)=\text { base_pen } \times(1+\text { inflation })^{\lfloor i / 12\rfloor}
$$

where base_pen is the penalty per unit product before inflation. 
5. The Total Flow Costs $(T F C)$

This term represents the net expenditure on the feed of raw material to the reactor and is given by:

$$
T F C=\sum_{i=1}^{N M} \sum_{j=1}^{4} \operatorname{cof}(i, j) \times f f r(i, j)
$$

where $\operatorname{cof}(i, j)$ is the cost of raw material per unit volume per week for week $j$ of month $i$, adjusted for inflation at that time:

$$
\operatorname{cof}(i, j)=\text { base_cof } \times(1+\text { inflation })^{\lfloor i / 12\rfloor}
$$

where base_cof is the cost of raw material per unit volume per week before inflation.

If the Net Costs are represented by $N C$, the objective function for this optimisation problem takes the form:

$$
\min N C=-G R S+T I C+T C C C+N P U D+T F C
$$

The essential elements of the problem formulation have now been described in detail. The aim is to make the appropriate decisions in order to minimise the net costs (or maximise the net profit) of the industrial process, when subject to the process model, initial and junction conditions and the constraints. It is highlighted that the catalyst changeover decision variables (y) occur linearly in all elements of the problem formulation. Thus, these variables are expected to exhibit a bang-bang behaviour in the optimal solution and the constraint, $y(i) \in[0,1]$ is equivalent to $y(i)=\{0,1\}$.

In the next sections, the problem solution implementation details will be discussed and the results obtained will be presented. As will be seen, the complex nature of the problem caused complications in obtaining solutions using the solvers currently available. Different solution implementations were 
attempted on different solvers: Implementation I was performed on MATLAB and Implementation II was carried out in Python, each of which had their own relative advantages.

The elements of the problem set up here are similar to that in Houze et al. (2003) and Bizet et al. (2005). However, those publications did not reveal any parameters used in their studies, citing confidentiality reasons. So, in this article, case studies were created using a set of constructed parameter values, which have been mentioned in Table B.7. The time horizon chosen here is 3 years, which is more realistic in present day industries compared to the much longer duration studied in Houze et al. (2003).

The problem size details for the chosen time horizon, applicable for all case studies, are shown in Table B.8. It is important to note that the number of variables and constraints in this formulation are much smaller than if MINLP approaches were used.

\subsection{Problem solution implementation I, results and discussions}

\subsubsection{Implementation I details}

Implementation I was performed on MATLAB ${ }^{\circledR}$ R2018a with its Optimisation Toolbox ${ }^{\mathrm{TM}}$ (MATLAB and Optimisation Toolbox, 2018), as a code that solves a standard multistage optimal control problem using the feasible path approach, by linking an ODE solver with the optimiser fmincon. Two types of ODE solvers were tried: the ode15s solver available on MATLAB ${ }^{\circledR}$ R2018a (Shampine and Reichelt, 1997) and the IDAS solver of sundialsTB, a MATLAB interface to the open-source set of differential-algebraic equation solvers, SUNDIALS (Serban, 2009). In both cases, the solver was designated to have an absolute tolerance of $10^{-6}$ and a relative tolerance of $10^{-4}$. The Jacobian was provided to the solvers to improve its reliability and efficiency. It was found that $I D A S$ of sundialsTB was faster in computation compared 
to ode15s and so was preferred for this implementation.

The optimisation on fmincon was performed using the Sequential Quadratic Programming (SQP) algorithm (Nocedal and Wright, 2006) with the following convergence criteria: constraint tolerance of $10^{-3}$, step tolerance of $10^{-3}$ and optimality tolerance of $10^{-4}$. A forward finite difference scheme was used for the estimation of gradients. Given the wide variation in the magnitude of the different decision variables (e.g. $y \in[0,1]$, but sales $\sim 10^{3}$ ), the starting points to the optimiser were scaled down using the respective upper bounds of each decision variable to avoid scaling problems in the optimisation. Further, in order to accelerate convergence, constraint (37) was scaled down by a factor of $10^{3}$ and the objective function value was scaled down by a factor of $10^{6}$.

In order to demonstrate the robustness of the developed methodology, it was desired to obtain a solution from a set of random values for the initial guesses of the decision variables to the optimiser. However, it was important to ensure that the set of random starting points were a set of 'feasible' points. Using highly infeasible starting points in this problem of complex nature could cause great difficulties to the optimiser in converging to a solution.

So in the initial part of Implementation I called Phase 1, a set of feasible start points for the decision variables was obtained by first generating a set of random points using the rand function in MATLAB ${ }^{\circledR}$ and running the optimisation model with the objective function set to zero. These feasible points were then used as the starting values for the actual optimisation problem in Phase 2 of the implementation. An algorithmic flowchart for Implementation I is shown in Figure 2.

The implementation was performed on a $3.2 \mathrm{GHz}$ Intel Core i5, $16 \mathrm{~GB}$ RAM, Windows machine running on Microsoft Windows 7 Enterprise. Since 


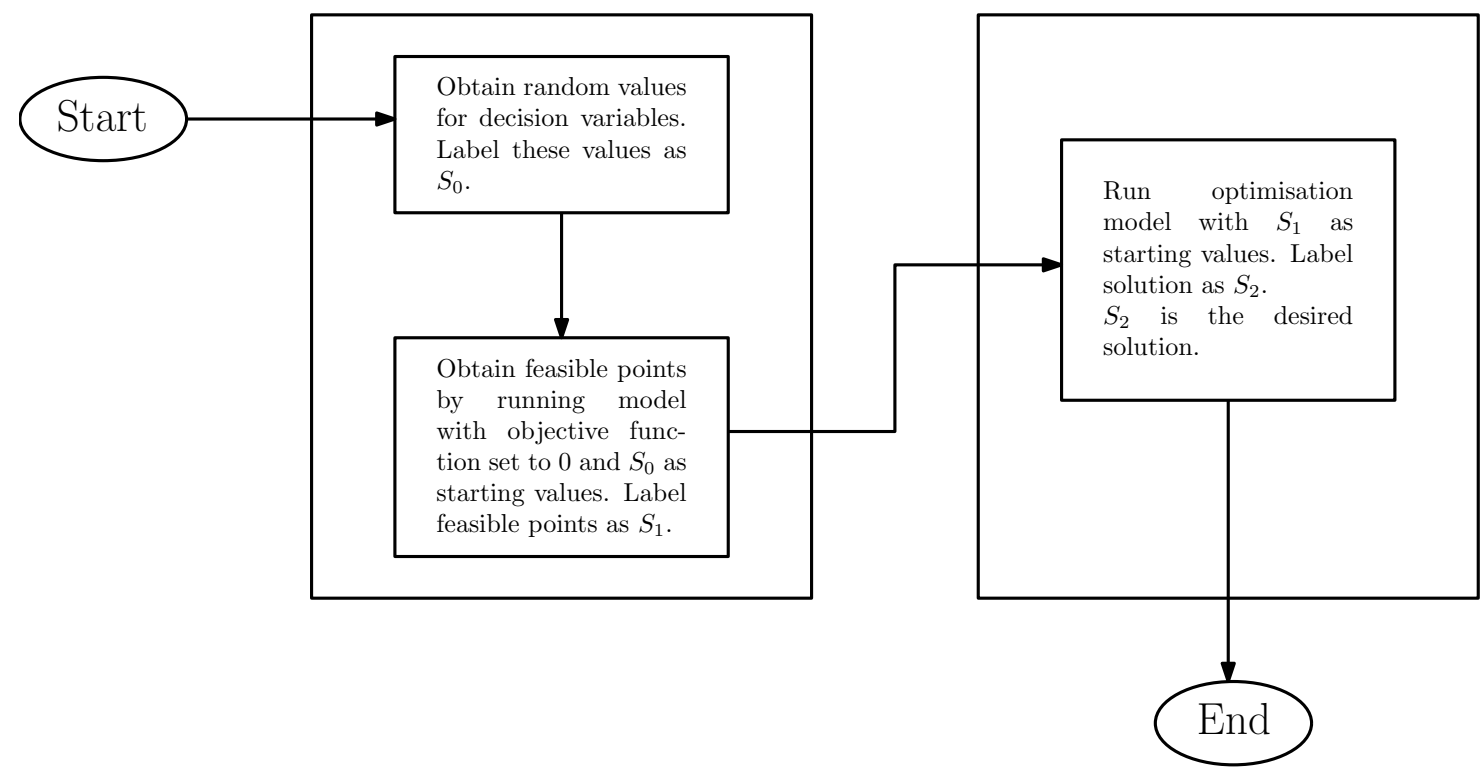

Figure 2: An algorithmic flowchart for Implementation I

the problem is non-convex, multiple runs were performed with different starting points. Test runs were performed using the Parallel Computing Toolbox ${ }^{\mathrm{TM}}$ on MATLAB ${ }^{\circledR}$ to compare the computational times between parallelising the gradient evaluations versus parallelisation of a loop of multiple start points using a parfor loop, and the latter was found to be faster. So, using the parfor loop for parallelisation, 50 runs were attempted for each case study.

\subsubsection{Implementation I: General performance discussion}

It was found that Implementation I had limited success when applied to Case Studies A and B whereas for Case Studies C and D, the technique failed completely. While some runs in Case Studies A and B exhibited a very good bang-bang behaviour for the catalyst changeover controls, in many other simulations, the runs either converged prematurely to poor solutions or crashed due to the integrator failing (Table B.9). Statistics regarding the solutions obtained and the computational effort involved, for the successful runs of 
Case Studies A and B are given in Tables B.10 and B.11, respectively. For Case Studies C and D, every single run crashed showing an error with the integration. These unexpected integration problems were experienced by both sets of ODE solvers which were tried. These problems could probably be attributed to the inadequacies of the MATLAB ODE suite in integrating the more nonlinear differential equations of Case Studies C and D.

Overall, the performance of Implementation I was unsatisfactory in providing solutions to all case studies. Despite this, there is a very good reason for reporting this solution procedure in this article: it is observed that a bang-bang behaviour is exhibited by the catalyst changeover controls, even when those linear controls occur in combination with other process control variables that occur nonlinearly in the system equations. This is consistent with the predictions of the Pontryagin analysis done in Appendix A. In the ensuing text, the optimal control and state variables of the most profitable run from the set of 50 different, random starting points for each of Case Studies A and B are reported, along with relevant economic statistics.

\subsubsection{Case Study A: Results and discussions}

Figures $3-6$ and Table 1 report the features of the best local optimum among the 13 successful runs for Case Study A, in which the main reaction is of first order kinetics with respect to the reactant and the catalyst deactivation kinetics is independent of the species' concentrations.

Figure 3 illustrates the variation of the monthly catalyst changeover controls over the whole time horizon. It can be seen that these controls take values of either 0 or 1 , thus exhibiting a bang-bang behaviour, consistent with the prediction for linear controls from the analysis in Appendix A. The graph indicates that the optimal policy for the industrial process is to use 4 of the 6 available catalysts over the 3-year horizon, with the 3 replacements $(y=0)$ occurring on the $8^{\text {th }}, 17^{\text {th }}$ and $24^{\text {th }}$ months. The first replacement 


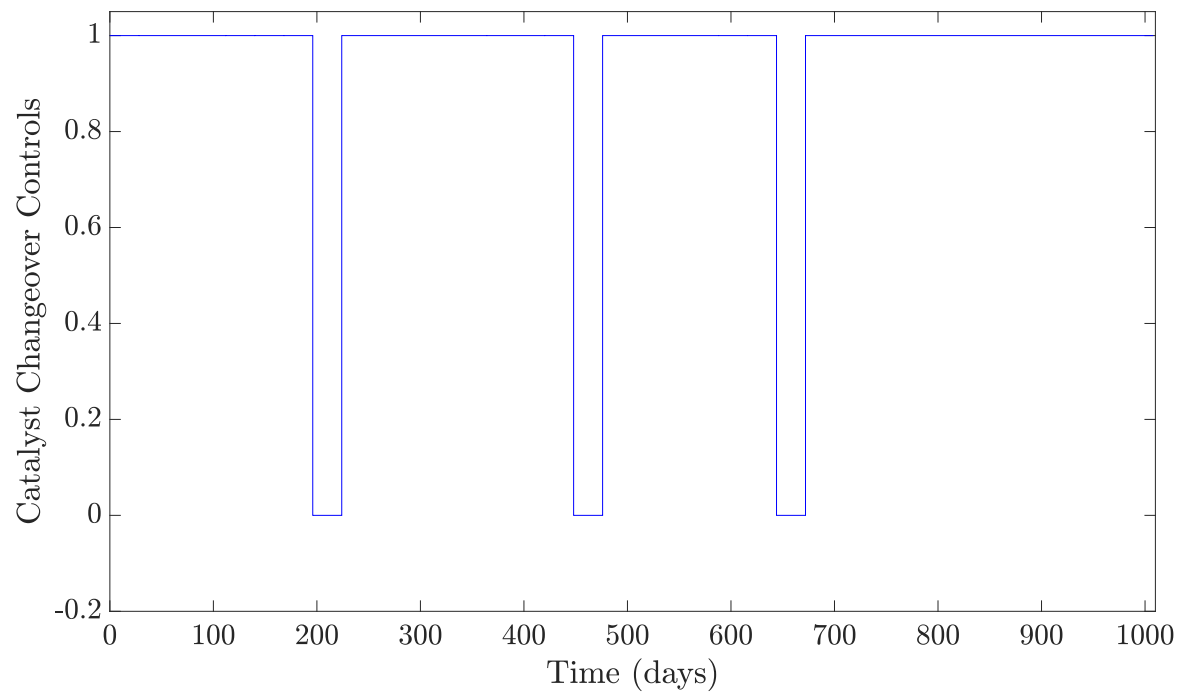

Figure 3: The variation of the catalyst changeover controls over the time horizon for Case Study A

occurs during the quarter of lowest demand in order to minimise losses. The other replacements occur only when a sufficient inventory level (Figure 6) is present to meet the demand during process shut-down.

Figure 4 plots the weekly flow rates to the reactor $(f f r)$ and temperatures of operation $(T)$, made dimensionless by their respective upper bounds and the exit concentration of the reactant from the reactor $(c R)$, over the whole time horizon of the process. Some notable points regarding these trends:

- The model's optimal policy during catalyst operation is to maintain a constant exit conversion by reducing the flow rate to compensate for the catalyst deactivation and operate temperature at its upper bound. This is consistent with the work of Szépe and Levenspiel (1968) for continuous reactors, which predicted similar policies when the main reaction is more sensitive to temperature than the catalyst deactivation and the latter is independent of the species' concentration. 


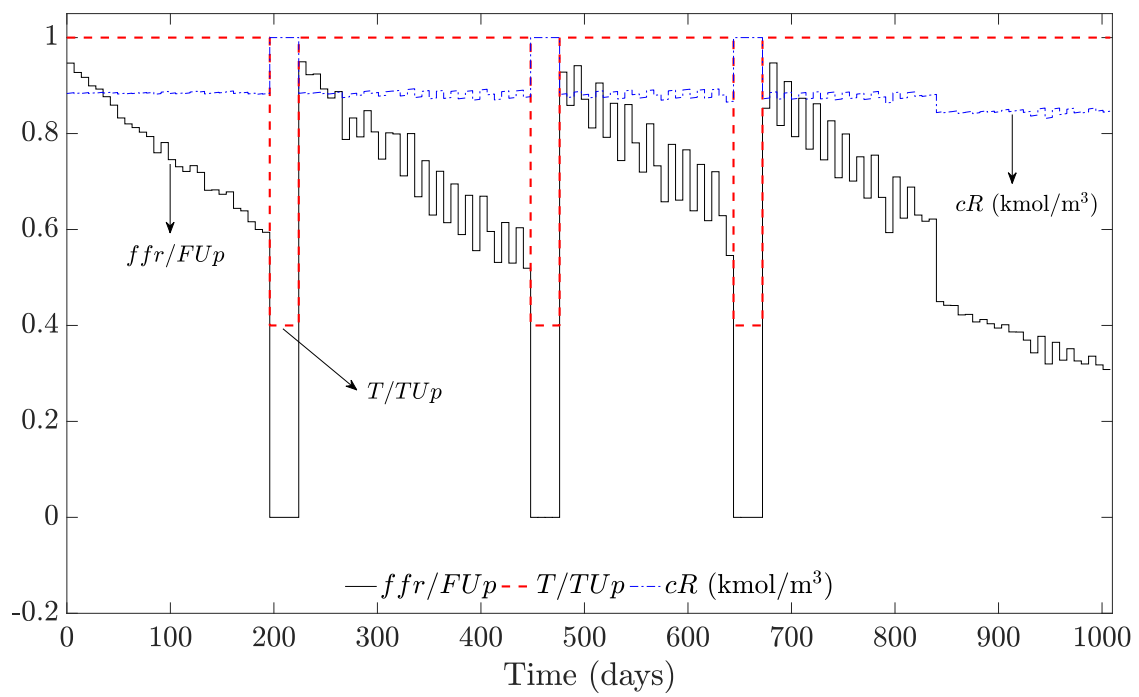

Figure 4: The variation of the feed flow rate, temperature and reactant exit concentration over the time horizon for Case Study A

- During the operation of the last catalyst, the sharp drop in the flow rate causes a corresponding effect in the exit concentration and this occurs to bring the production rate to a value that exactly fulfils the demand for the remainder of the time horizon.

- It is highlighted that the flow rate does not exhibit a bang-bang behaviour as these controls appear nonlinearly in the system equations, consistent with the prediction from Appendix A. It is interesting to note that the temperature controls only take values at their upper or lower bounds, and this follows from the nature of the problem and the constraints imposed, without a correlation to their nonlinear occurrence in the system equations.

A comparison of the optimal quantity of product sales with the corresponding product demand and unmet demand for each week over the whole time horizon, is shown in Figure 5. While a considerable amount of unmet demand exists during the first year of the process, it is nil for the remainder 
years. Given that the product sales price increases annually due to inflation, a greater amount of profit can be obtained by selling more product during later years and so the model prefers to sell less during the first year and more in the later years. It is also highlighted that the sales continue throughout the time horizon, even at times of process shut down for catalyst replacement. Taking inflation into account, the model operates the sales in an efficient manner such that the inventory level (Figure 6) is adjusted to balance the trade-offs between storing a sufficient quantity of product to meet seasonal demand and high storage costs.

The variation of the catalyst activity, catalyst age, inventory level and cumulative inventory costs over the time horizon are shown in Figure 6. It is highlighted that towards the end of the first year, the inventory level shows a significant increase, despite there being a considerable amount of unmet demand at that time. This happens in order to enable greater amount of sales during later times when the product sales price has increased due to inflation, thereby enlarging the profit obtained.

The magnitudes of the various economic aspects that form the elements of the objective function are given in Table 1 . The table indicates that the cost of flow and raw material constitutes more than half of the total expenses with the net penalty for unmet demand also forming a significant proportion. The cost of catalyst changeovers contributes relatively less while the inventory costs form a very low percentage of the total expenditure. It is also seen that the costs of operation take away about $43.6 \%$ of the revenue generated by the product sales. 
(a)

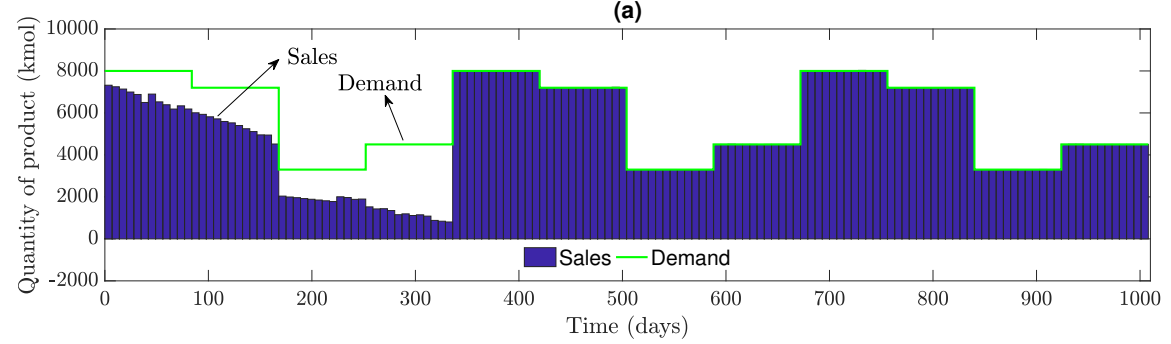

(b)

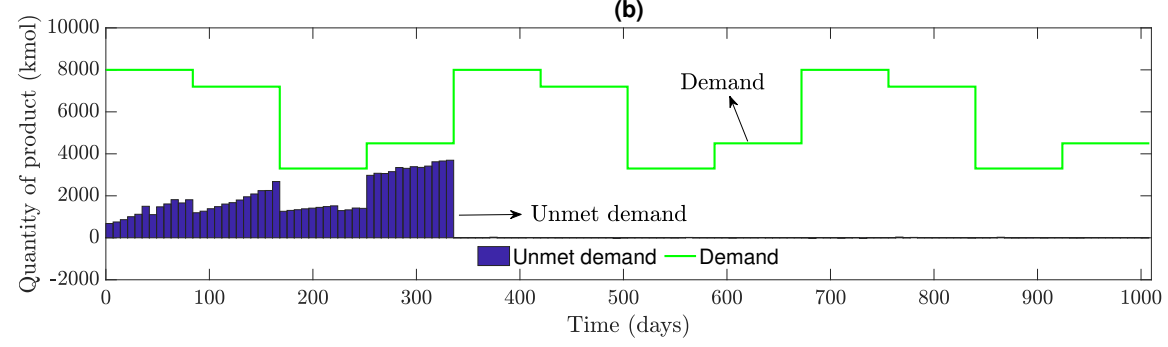

Figure 5: The variation of (a) sales and (b) unmet demand, in comparison to the demand over the time horizon for Case Study A

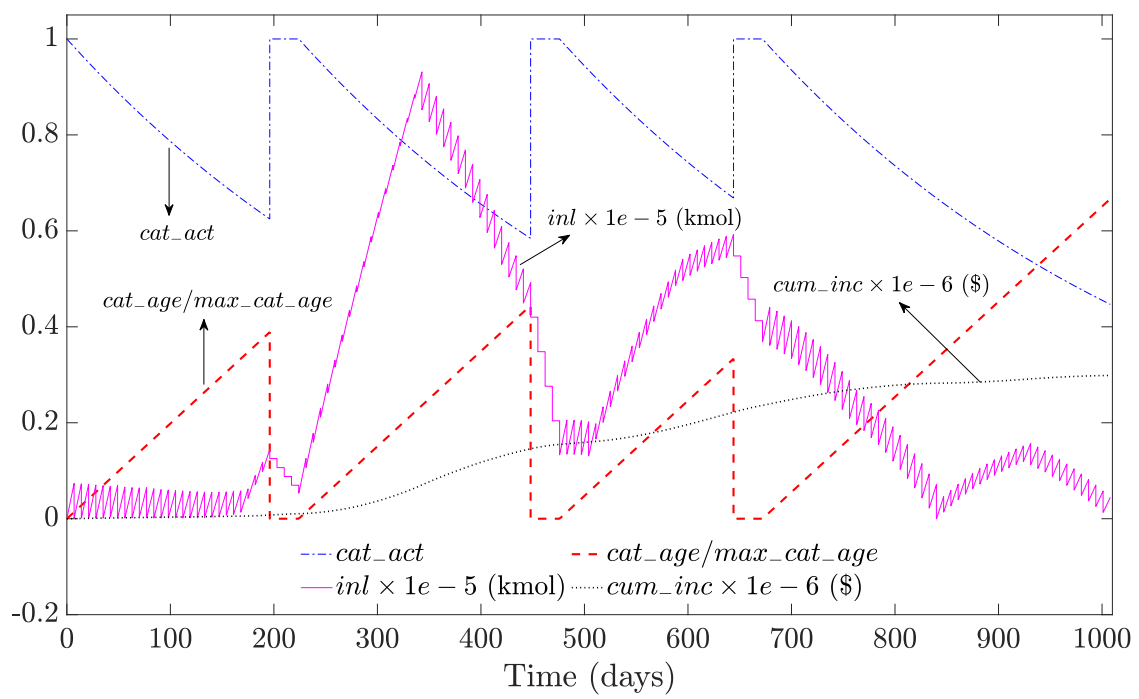

Figure 6: The variation of the catalyst activity, catalyst age, inventory level and cumulative inventory cost over the time horizon for Case Study A 
Table 1: Economic aspects of the best solution of Case Study A

\begin{tabular}{|c|c|c|c|}
\hline \multicolumn{2}{|c|}{ Economic aspect } & Symbol & Value (\$ Millions) \\
\hline \hline \multirow{3}{*}{ Costs } & Gross Revenue from Sales & $G R S$ & 776.422 \\
\cline { 2 - 4 } & Total Inventory Costs & TIC & 0.299 \\
\cline { 2 - 4 } & Total Costs of Catalyst Changeovers & TCCC & 30.999 \\
\cline { 2 - 4 } & Net Penalty for Unmet Demand & NPUD & 117.089 \\
\cline { 2 - 4 } & Total Flow Costs & TFC & 189.955 \\
\hline \hline \multicolumn{2}{|c|}{ Profit } & $-N C$ & 438.08 \\
\hline
\end{tabular}

\subsubsection{Case Study B: Results and discussions}

Figures $7-10$ and Table 2 report the features of the best local optimum among the 22 successful runs for Case Study B, in which the main reaction is of first order kinetics with respect to the reactant and the catalyst deactivation kinetics is proportional to the reactant concentration.

Figure 7 shows the variation of the monthly catalyst changeover controls over the time horizon. Once again, a bang-bang behaviour is exhibited, consistent with the analysis in Appendix A. The recommendation is to use 4 of the 6 available catalysts over the 3 -year horizon, with the 3 replacements $(y=0)$ occurring on the $9^{\text {th }}, 16^{\text {th }}$ and $23^{\text {rd }}$ months. Once again, the first replacement occurs at a time to minimise losses and the other changeovers occur only when there is sufficient inventory to meet the demand.

Figure 8 is the analogue of Figure 4 in Case Study A. The trends of $f f r$ and $c R$ during catalyst operation are different from in Case Study A: the decrease in $f f r$ is such that its rate of decrease is slower than the rate of catalyst deactivation and this causes $c R$ to show a roughly linear increase in 


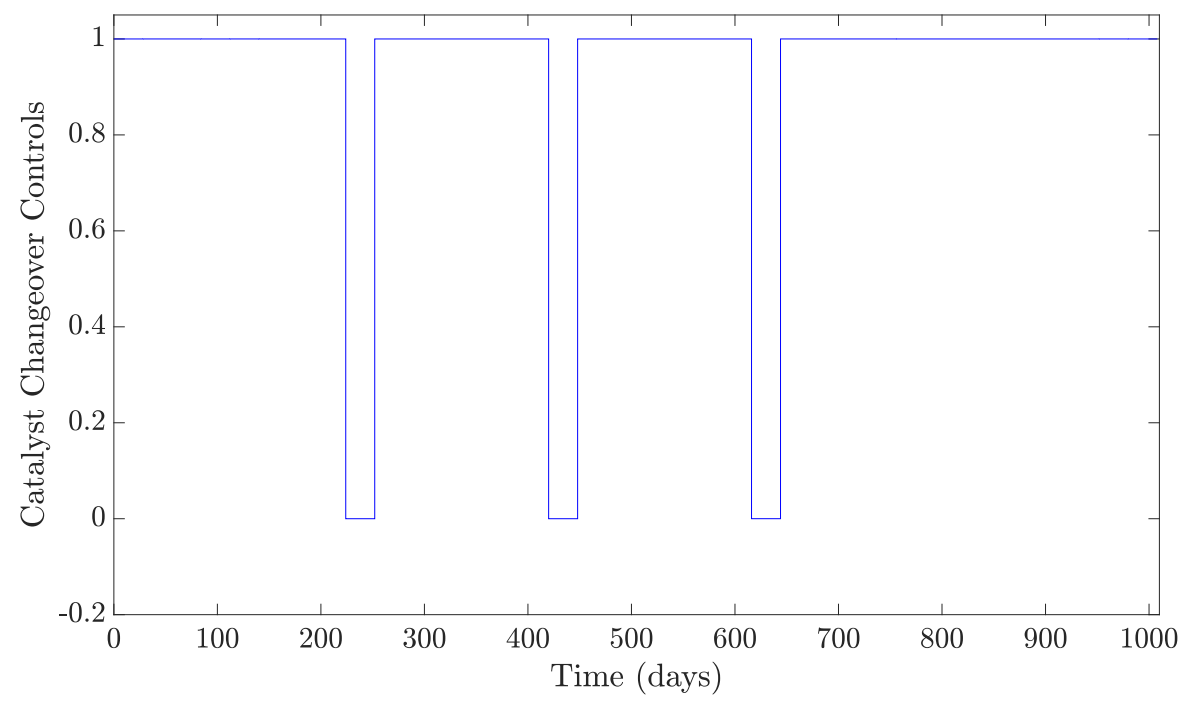

Figure 7: The variation of the catalyst changeover controls over the time horizon for Case Study B

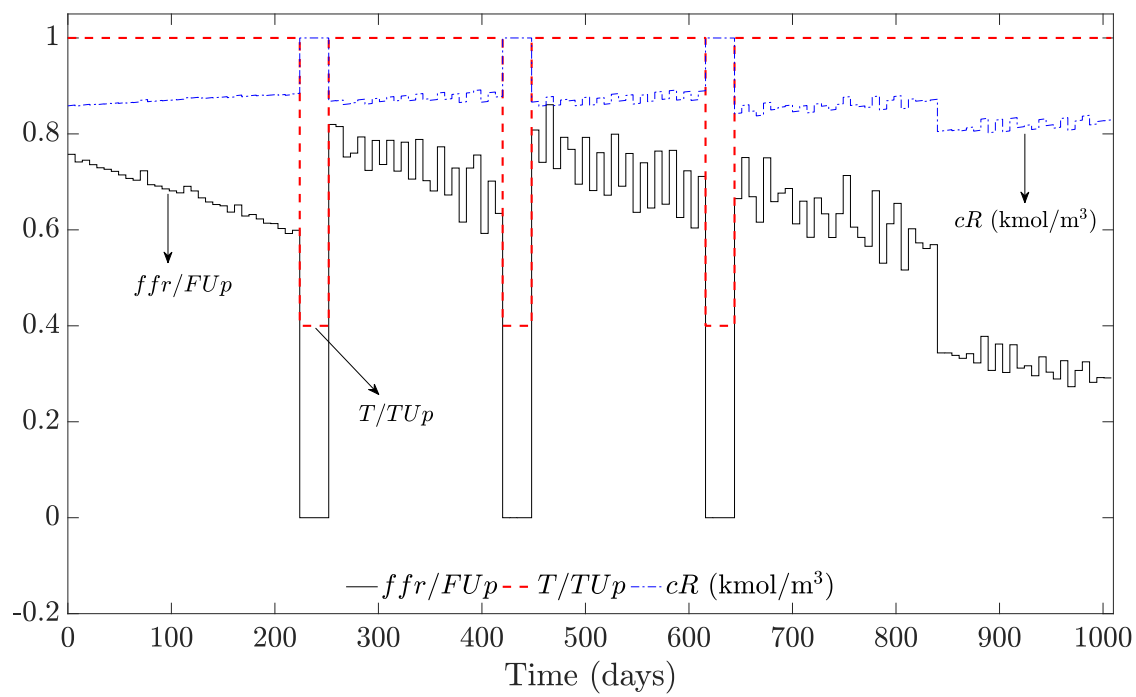

Figure 8: The variation of the feed flow rate, temperature and reactant exit concentration over the time horizon for Case Study B 
magnitude. This behaviour is not consistent with the work of Crowe (1976) which predicted maintaining a constant exit conversion as the optimal policy at the reactor level, even when the catalyst deactivation kinetics is dependent on the reacting species' concentration. An explanation for this profile of $c R$ is offered using the following points:

- A larger magnitude of $c R$ implies a faster deactivation of the catalyst, following from Equation (10), and this is unfavourable for the process.

- A larger magnitude of $c R$ means a larger reaction rate, following from Equation (13), and this is favourable for the process.

Thus, there is a trade-off to be balanced in maintaining a particular magnitude of $c R$. The flow rate is chosen such that at the beginning of operation of a new catalyst, a relatively low value of $c R$ occurs, which although lowers the reaction rate, it prevents the fresh catalyst from deactivating too fast. However, as the catalyst deactivates, the focus shifts to maintaining a higher reaction rate and this is done by the appropriate reduction of $f f r$ to raise $c R$. This linearly increasing trend enables to optimally balance the positive and negative effects of maintaining a particular magnitude of $c R$.

Figures 9 - 10 and Table 2 are the analogues of Case Study B to Figures 5 - 6 and Table 1 in Case Study A. The profile for the catalyst activity during catalyst operation in Figure 10 follows from equation (10). The explanations for the trends of all other variables in Figures 9 and 10 are similar to those of their Case Study A analogues. Table 2 shows that the costs of operation take away about $39.5 \%$ of the revenue generated by the product sales. 
(a)

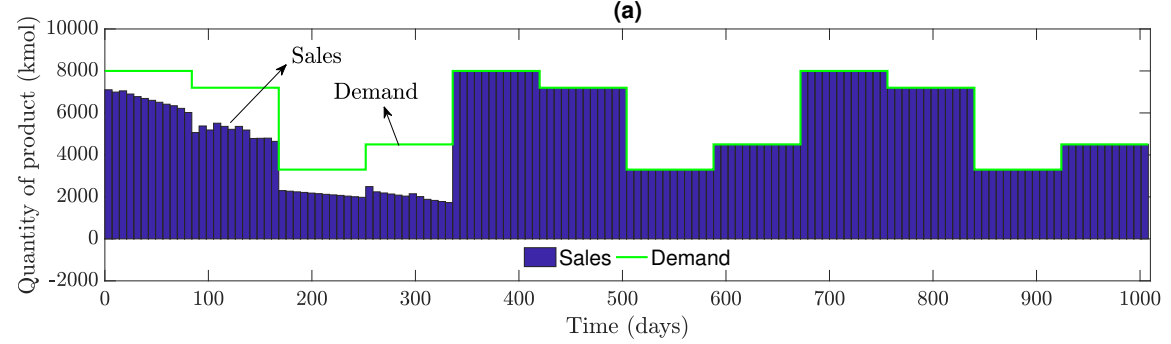

(b)

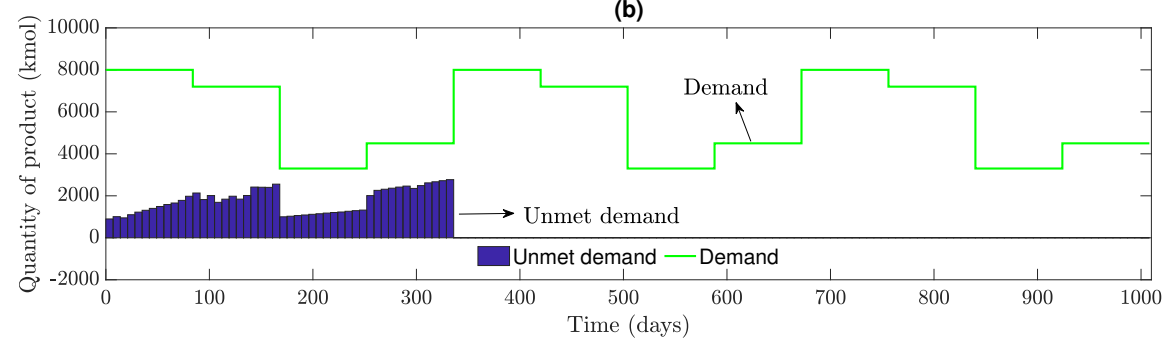

Figure 9: The variation of (a) sales and (b) unmet demand, in comparison to the demand over the time horizon for Case Study B

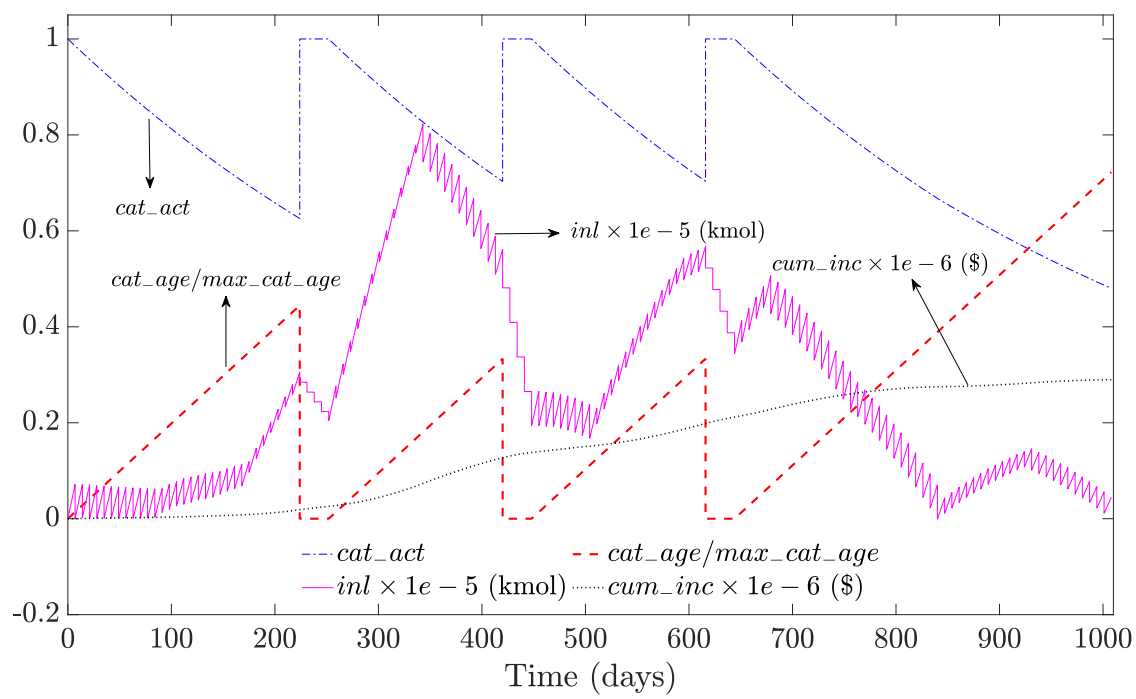

Figure 10: The variation of the catalyst activity, catalyst age, inventory level and cumulative inventory cost over the time horizon for Case Study B 
Table 2: Economic aspects of the best solution of Case Study B

\begin{tabular}{|c|c|c|c|}
\hline \multicolumn{2}{|c|}{ Economic aspect } & Symbol & Value (\$ Millions) \\
\hline \hline \multirow{3}{*}{ Costs } & Gross Revenue from Sales & $G R S$ & 785.245 \\
\cline { 2 - 4 } & Total Inventory Costs & TIC & 0.290 \\
\cline { 2 - 4 } & Total Costs of Catalyst Changeovers & TCCC & 30.999 \\
\cline { 2 - 4 } & Net Penalty for Unmet Demand & NPUD & 106.061 \\
\cline { 2 - 4 } & Total Flow Costs & TFC & 172.67 \\
\hline \hline \multicolumn{2}{|c|}{ Profit } & $-N C$ & 475.225 \\
\hline
\end{tabular}

\subsection{Problem solution implementation II, results and discussions}

Given the inadequacies of Implementation I, it was decided to attempt an alternate implementation in Python ${ }^{\mathrm{TM}}$ 3.7.1 under PyCharm 2018.2.4 (Community Edition). This section discusses the details and performances of a preliminary implementation called Implementation IIA, before doing the same for Implementation II, a modification of the former. Subsequently, the results of all case studies obtained using Implementation II are presented.

\subsubsection{Implementation IIA details}

Implementation IIA was carried out as a Python code that solved a standard multistage optimal control problem using the feasible path approach, similar to that of Implementation I. The code was written using CasADi, an open source software that enables a symbolic framework for numerical optimisation (Andersson, 2013). The elements of the problem, as given in Section 3.1, were defined as symbolic expressions using CasADi v3.4.5. The Automatic Differentiation (AD) feature of CasADi enabled constructions of symbolic expressions of the derivatives of all predefined functions, thereby maintaining differentiability to an arbitrary order. This allowed for an efficient calculation of gradients, that did not suffer from round-off and trunca- 
tion errors, unlike gradient calculation using finite differences.

CasADi contains plug-ins to the open source SUNDIALS suite (Hindmarsh et al., 2005) and IPOPT by COIN-OR (Wächter and Biegler, 2006), which were used for the integration of ODEs and optimisation, respectively. The IDAS solver of SUNDIALS was used for the integration of the ODEs with the following termination criteria: an absolute tolerance of $10^{-6}$ and a relative tolerance of $10^{-6}$. The optimisation by IPOPT had, respectively, the following termination and acceptable termination criteria: $10^{-4}$ and $10^{-4}$ for the optimality error, 1 and $10^{6}$ for the dual infeasibility, $10^{-4}$ and $10^{-2}$ for the constraint violation, and $10^{-4}$ and $10^{-2}$ for the complementarity. The acceptable number of iterations was set at 15 .

The above implementation procedure was run on the same hardware and operating system used for Implementation I. A set of random starting guesses for the decision variables were provided using the rand method of the random class within the numpy module.

\subsubsection{Implementation IIA: General performance discussion}

For multiple test runs, it was found that the catalyst changeover actions did not exhibit a bang-bang behaviour when this implementation methodology was used. Other adjustments such as tighter optimality tolerances, scaling of the objective functions and constraints or providing feasible starting guesses to the decision variables made little difference and there remained non-integral catalyst changeover control values in the final solution. Thus, the analysis done in Section 2 is not applicable here and further modifications were needed to Implementation IIA in order to attain the desired results and this led to Implementation II. 


\subsubsection{Implementation II details}

Implementation II is composed of executing Implementation IIA with a penalty term homotopy, a technique is similar to that suggested by Sager (Sager, 2005, 2009). The principle of this method is to add a monotonically increasing penalty term to the objective function in equation (48) and solve a series of OCPs of generic form:

$$
F_{k}: \min \left[N C+M_{k} \sum_{i=1}^{N M} y(i)[1-y(i)]\right]
$$

$$
k=1,2,3 \ldots
$$

The first problem $(k=1)$ in the series is designated a weight of $M_{1}=0$ and so the solution of $F_{1}$ is equivalent to the solution of Implementation IIA. The procedure of the method is to initialise problem $F_{k+1}$ with the solution of $F_{k}$ and increase the penalty term in the objective of $F_{k+1}$ by choosing a weight $M_{k+1}>M_{k}$. This procedure is repeated until iteration $K$ such that weight $M_{K}$ is large enough to force all catalyst changeover controls to take values of either 0 or 1 . For the choice of parameters used in the set of case studies investigated in this article, the weight is increased as per the following arithmetic progression:

$$
M_{k+1}=\left(2 \times M_{k}\right)+\left(5 \times 10^{7}\right)
$$

$$
M_{1}=0
$$

$$
k=1,2,3 \ldots
$$

Every iteration, $k$, will be referred to as a 'major iteration' in this article. This progression for increasing the weights was chosen arbitrarily, by trial and error. It should be mentioned that if the weight is increased too slowly, the computational time becomes large, while if it is increased too fast, the optimiser can fail to recognise a solution and continue iterations indefinitely. 
The implementation was performed on the same hardware as for Implementations I and IIA. Once again, multiple runs were performed with different starting points due to the non-convex nature of the problem. Test runs using the multiprocessing module in Python, to parallelise a loop of multiple start points, executed slower than when the runs were done serially. So for each case study, 50 runs were executed in a serial manner.

\subsubsection{Implementation II: General performance discussion}

It was found that Implementation II produced high quality solutions for all case studies. Not in a single run for any case study, regardless of the degree of nonlinearity of the process model, was any integration or convergence problem encountered.

Statistics regarding the solutions obtained from the 50 runs for all case studies using Implementation II are given in Table B.12. The range of optimal profit values obtained for Case Studies A and B were comparable to those obtained from the limited set of successful runs for the same case studies using Implementation I, thereby indicating that the answers obtained in these case studies created using invented parameters are indeed optimal. The table also indicates that the number of catalyst replacements were lower and the catalyst ages longer for this implementation in comparison to Implementation I. However, such comparisons were not possible for the runs of Case Studies C and D as Implementation I failed to produce solutions for those case studies. Statistics regarding the computational effort involved are given in Tables B.13 and B.14.

Overall, Implementation II was more reliable and robust, compared to Implementation I, in producing high quality solutions. Next, the results of the best solution obtained using this implementation from the set of 50 runs, for each of the case studies, are discussed along with other relevant statistics. 


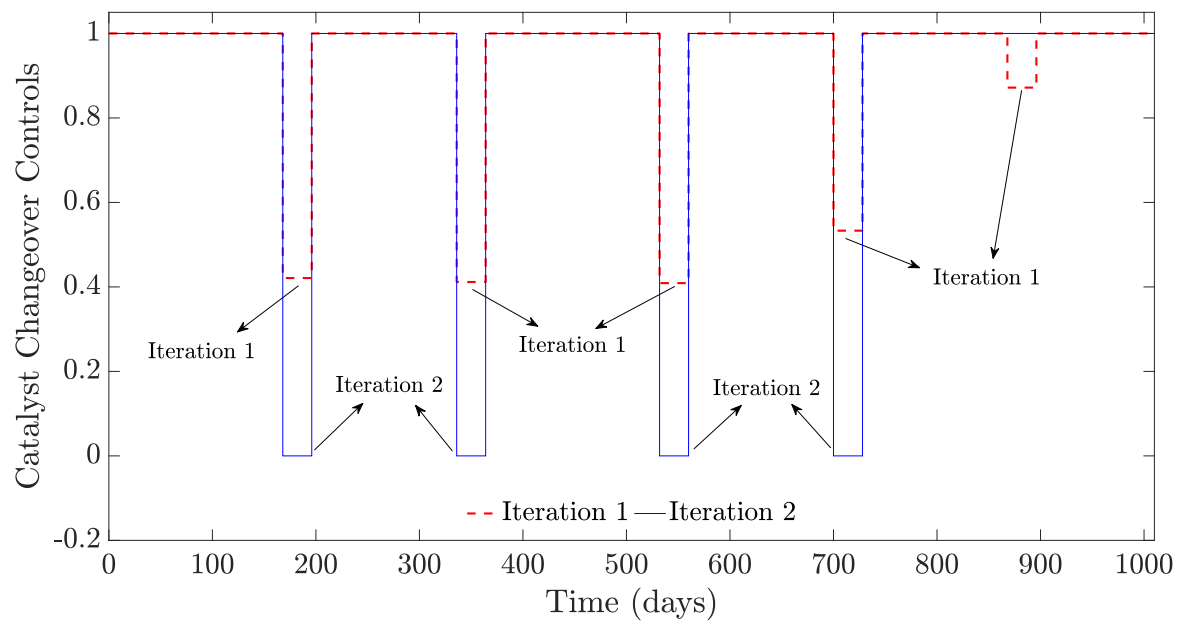

Figure 11: The variation of the catalyst changeover controls over the time horizon for Case Study A

\subsubsection{Case Study A: Results and Discussions}

Figures 11 - 14 and Table 3 report the features of the best local optimum among the 50 runs for Case Study A using Implementation II. These are the analogues of Figures $3-6$ and Table 1, respectively, obtained using Implementation I in Section 3.2.3.

Figure 11 shows the variation of the monthly catalyst changeover controls over the time horizon, across different major iterations. It is seen that the solution of the first major iteration is not of bang-bang form, while in the second iteration, integer values are obtained for these controls. The recommendation is to use 5 of the 6 available catalysts over the 3 -year horizon, with the 4 replacements $(y=0)$ occurring on the $7^{\text {th }}, 13^{\text {th }}, 20^{\text {th }}$ and $26^{\text {th }}$ months. Similar to Figure 3, the first replacement occurs at a time to minimise losses and the other replacements occur only when there is sufficient inventory to meet the demand. The other results presented are those obtained as solutions of the second major iteration. 


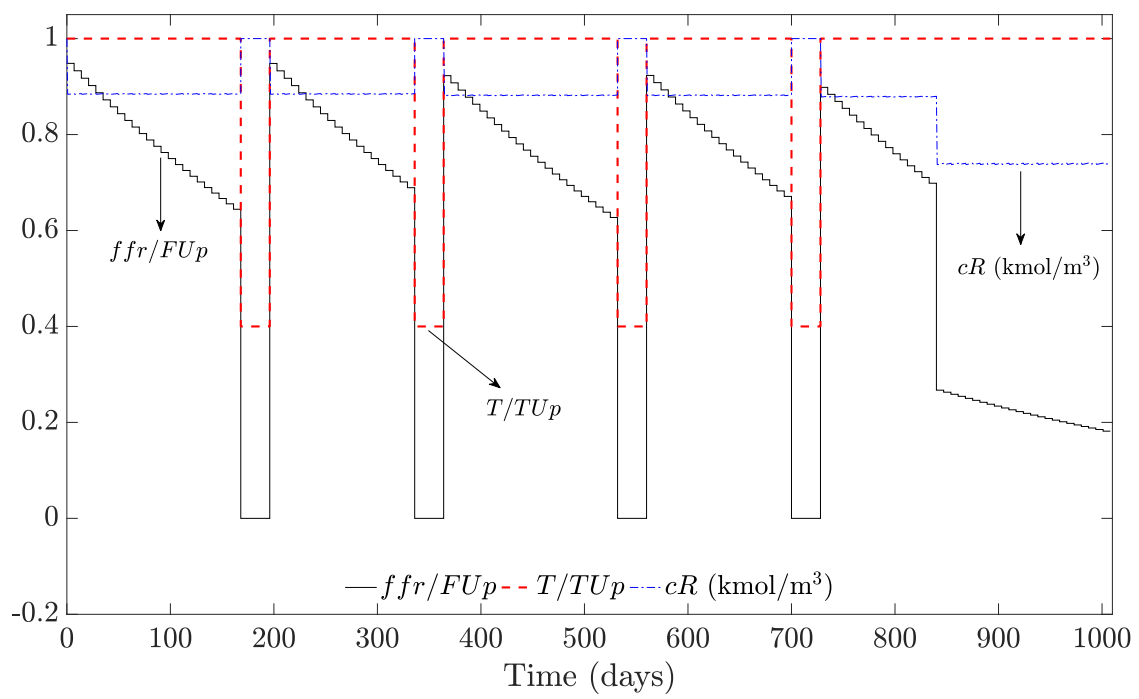

Figure 12: The variation of the feed flow rate, temperature and reactant exit concentration over the time horizon for Case Study A
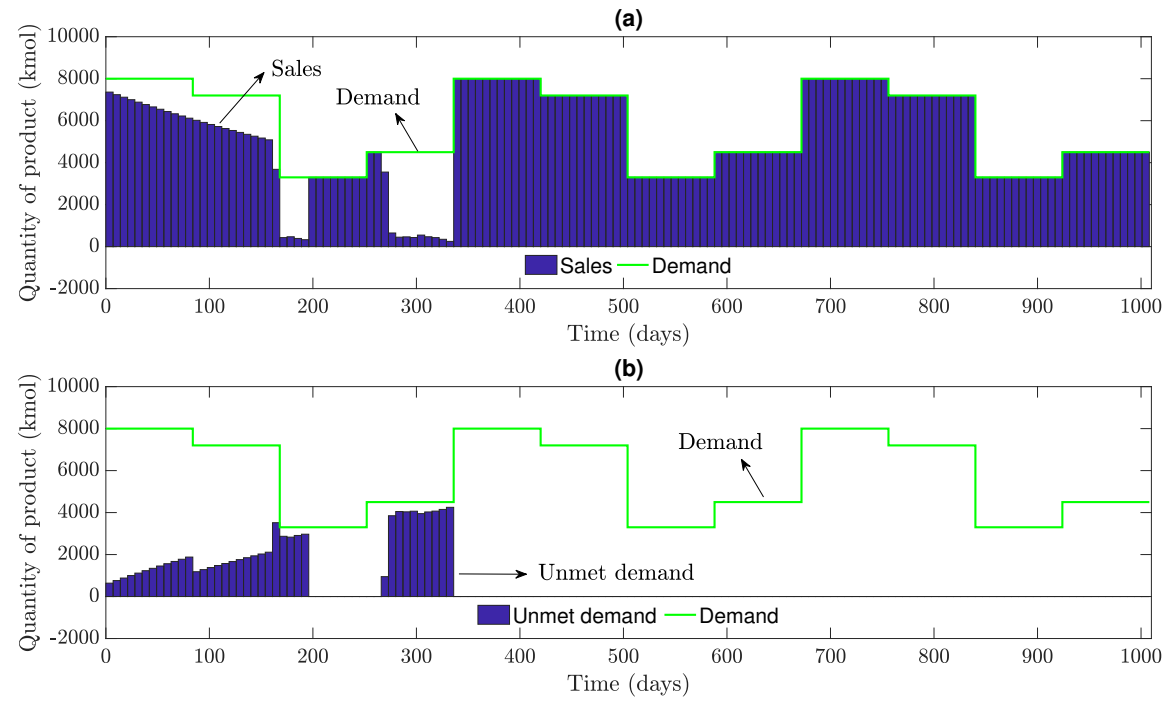

Figure 13: The variation of (a) sales and (b) unmet demand, in comparison to the demand over the time horizon for Case Study A 


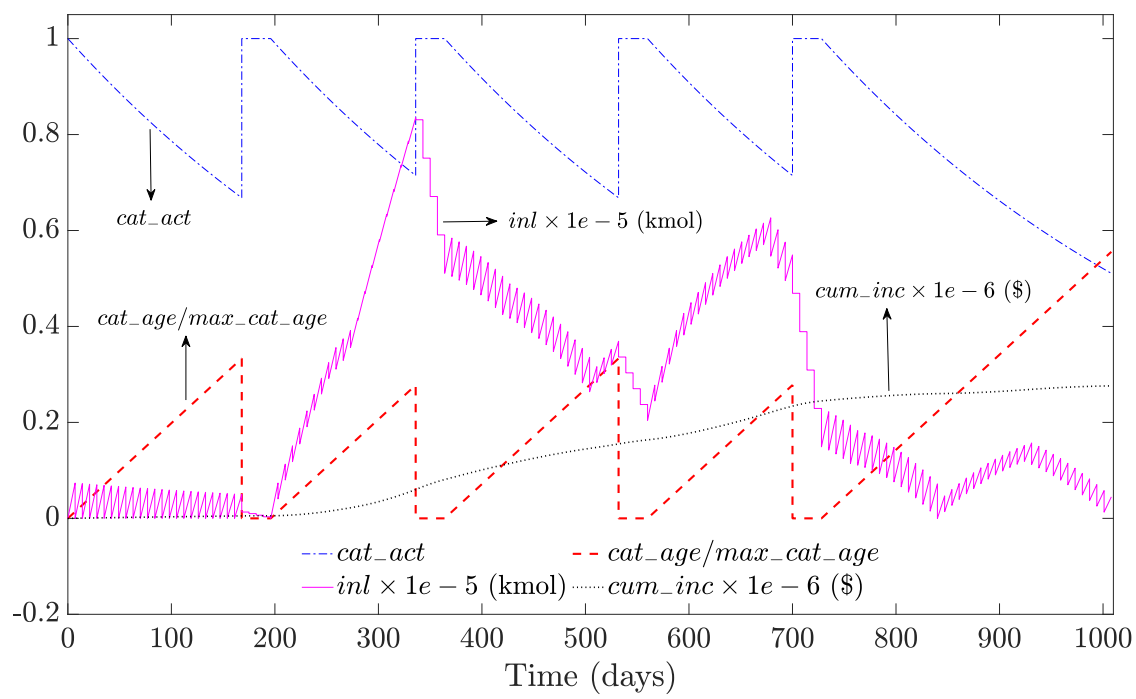

Figure 14: The variation of the catalyst activity, catalyst age, inventory level and cumulative inventory cost over the time horizon for Case Study A

Table 3: Details of the economic aspects for Case Study A

\begin{tabular}{|c|c|c|c|}
\hline \multicolumn{2}{|c|}{ Economic aspect } & Symbol & Value (\$ Millions) \\
\hline \hline \multirow{3}{*}{ Costs } & Gross Revenue from Sales & $G R S$ & 783.722 \\
\cline { 2 - 4 } & Total Inventory Costs & TIC & 0.276 \\
\cline { 2 - 4 } & Total Costs of Catalyst Changeovers & TCCC & 42.025 \\
\cline { 2 - 4 } & Net Penalty for Unmet Demand & NPUD & 107.96 \\
\cline { 2 - 4 } & Total Flow Costs & $T F C$ & 183.515 \\
\hline \hline \multicolumn{2}{|c|}{ Profit } & $-N C$ & 449.946 \\
\hline
\end{tabular}

The variation of the trends of variables in Figures 12 - 14 are similar to their analogues in Case Study A. Once again, the optimal policies suggested at the reactor level by Szépe and Levenspiel (1968) for continuous reactors are 
followed here for $c R$ and $T$. Table 3 shows that the profit here is comparable to that in Table 1.

\subsubsection{Case Study B: Results and Discussions}

Figures 15 - 18 and Table 4 report the features of the best local optimum among the 50 runs for Case Study B using Implementation II. These are the analogues of Figures 7 - 10 and Table 2, respectively, obtained using Implementation I in Section 3.2.4.

In this case, three major iterations are needed to force the catalyst changeover controls to take integer values (Figure 15) and the other results presented in this section correspond to the solution of the third major iteration. The explanations of the trends for all variables, and the final profit and costs values are very similar to those in Section 3.2.4.

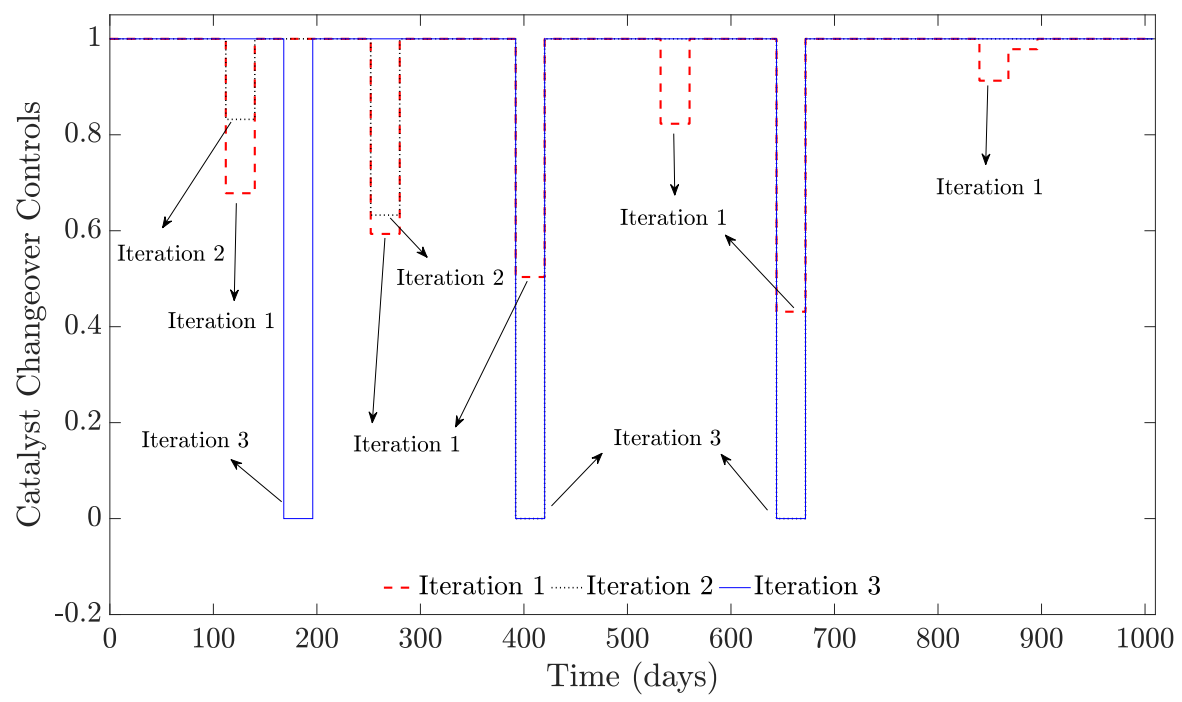

Figure 15: The variation of the catalyst changeover controls over the time horizon for Case Study B 


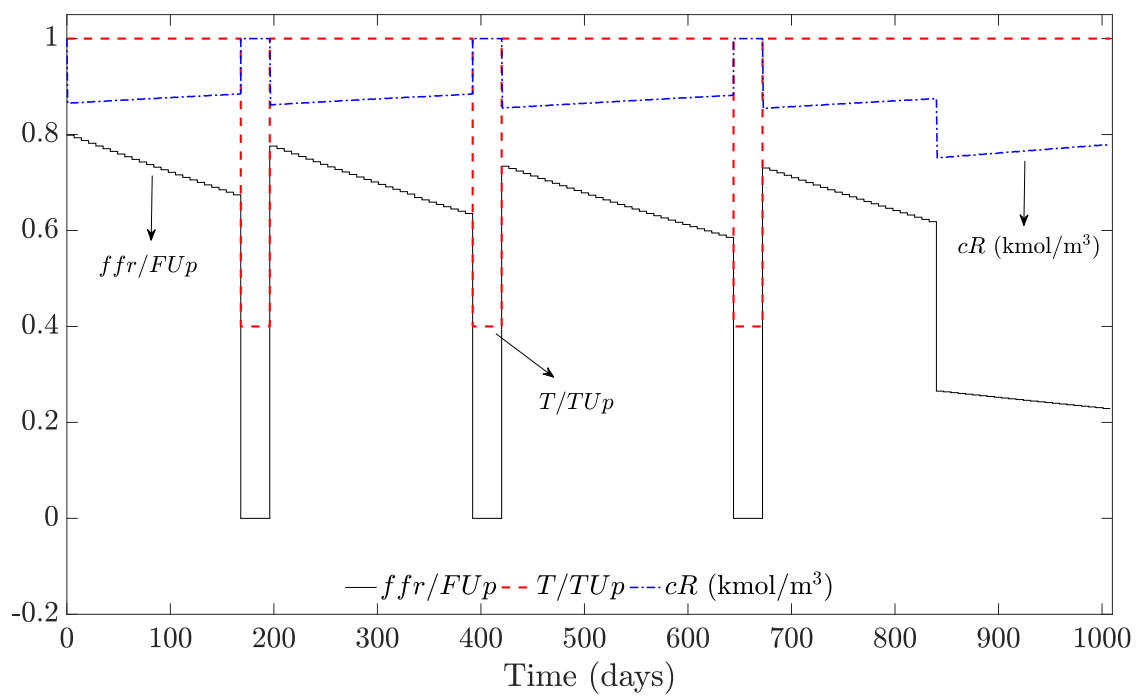

Figure 16: The variation of the feed flow rate, temperature and reactant exit concentration over the time horizon for Case Study B
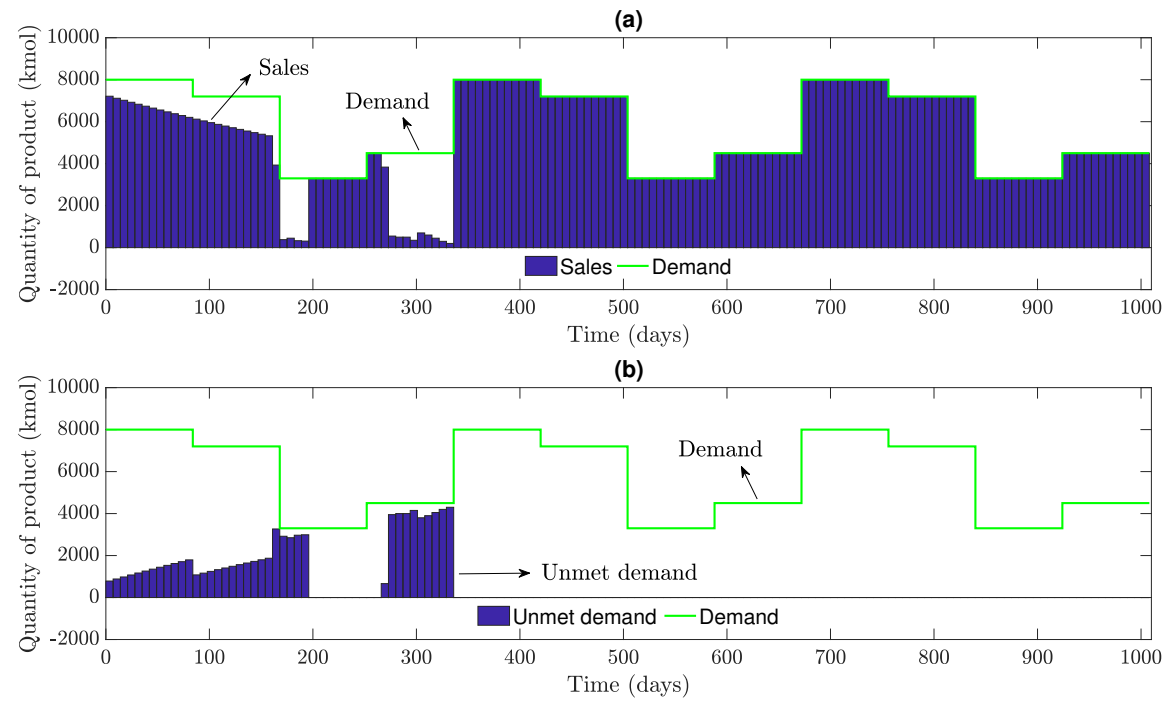

Figure 17: The variation of (a) sales and (b) unmet demand, in comparison to the demand over the time horizon for Case Study B 


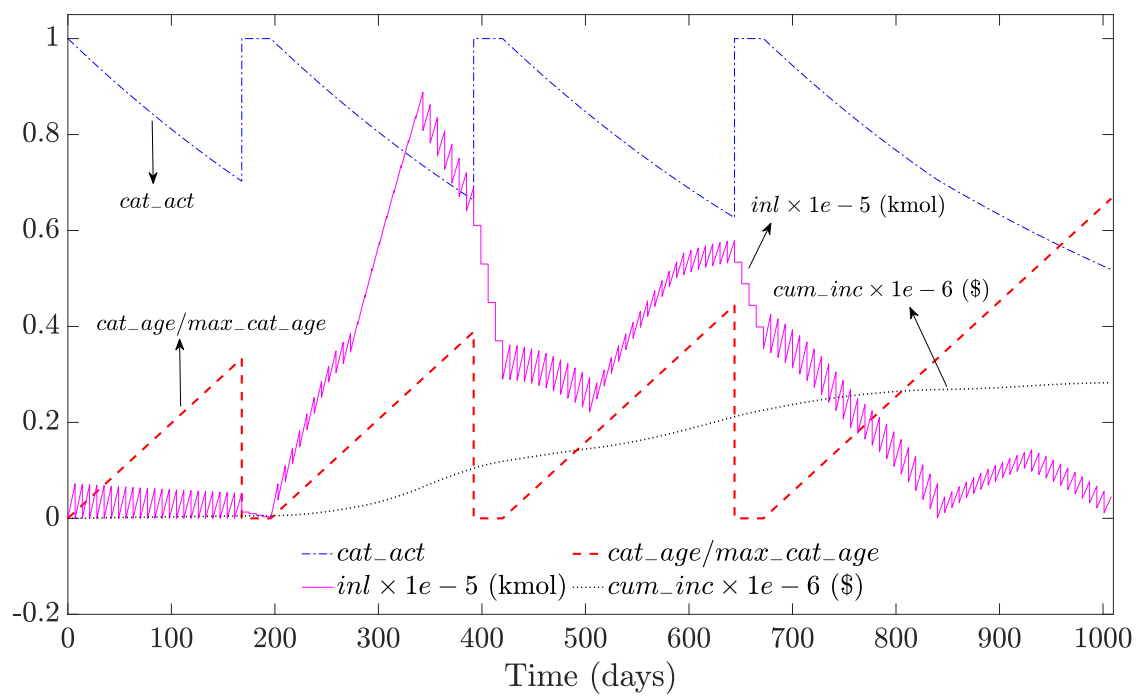

Figure 18: The variation of the catalyst activity, catalyst age, inventory level and cumulative inventory cost over the time horizon for Case Study B

Table 4: Details of the economic aspects for Case Study B

\begin{tabular}{|c|c|c|c|}
\hline \multicolumn{2}{|c|}{ Economic aspect } & Symbol & Value (\$ Millions) \\
\hline \hline \multirow{3}{*}{ Costs } & Gross Revenue from Sales & $G R S$ & 785.902 \\
\cline { 2 - 4 } & Total Inventory Costs & TIC & 0.282 \\
\cline { 2 - 4 } & Total Costs of Catalyst Changeovers & TCCC & 30.999 \\
\cline { 2 - 4 } & Net Penalty for Unmet Demand & NPUD & 105.235 \\
\cline { 2 - 4 } & Total Flow Costs & $T F C$ & 169.251 \\
\hline \hline \multicolumn{2}{|c|}{ Profit } & $-N C$ & 480.135 \\
\hline
\end{tabular}

\subsubsection{Case Study C: Results and Discussions}

Figures 19 - 22 and Table 5 report the features of the best local optimum among the 50 runs for Case Study C using Implementation II. Here the main reaction is of first order kinetics with respect to the reactant and 


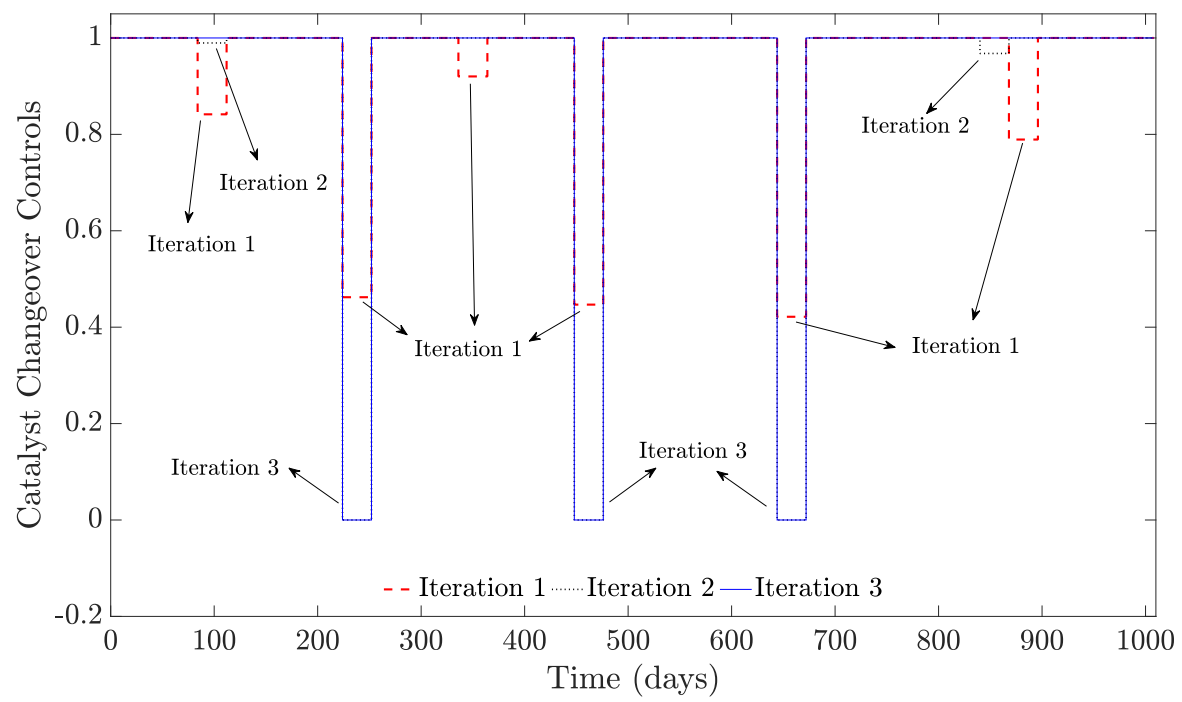

Figure 19: The variation of the catalyst changeover controls over the time horizon for Case Study C

the catalyst deactivation kinetics is dependent on the product concentration. Implementation I failed to obtain results for this case study, due to problems in integrating the highly nonlinear system of ODEs.

Figure 19 shows the variation of the monthly catalyst changeover controls over the time horizon, across different major iterations. In this case, three major iterations are needed to force the catalyst changeover controls to take integer values. 4 of the 6 available catalysts are used, with the changeovers occurring on the $9^{\text {th }}, 17^{\text {th }}$ and $24^{\text {th }}$ months, which are times when a sufficient inventory level is present to meet the demand. All other results presented here are those obtained at the end of the third major iteration.

Figure 20 shows that the profiles of $f f r$ and $c R$ during times of catalyst operation are different from other case studies and once again, the trend for $c R$ is not consistent with the work of Crowe (1976). The scenarios are: 


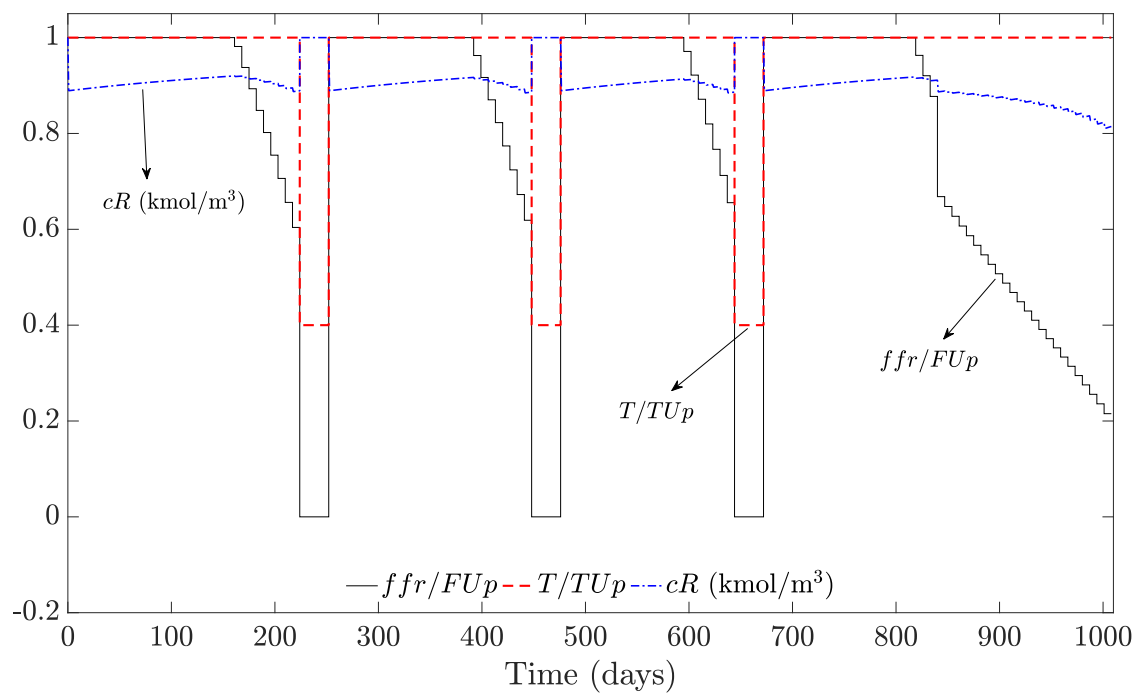

Figure 20: The variation of the feed flow rate, temperature and reactant exit concentration over the time horizon for Case Study C

- The $f f r$ is constant at its maximum value during when the deactivation of the catalyst causes $c R$ to increase with time.

- The $f f r$ decreases at a rate that causes $c R$ to decrease.

The flow costs are high in the former scenario while they are considerably lower in the latter. However, a higher value of $c R$ in the former scenario is favourable economically as this leads to a slower rate of catalyst deactivation and a larger reaction rate, following from equations (11) and (13), respectively, while the reverse is true in the latter scenario.

Thus, it can be said that there is an interplay between the elements of the process economics, which affect the variation of $f f r$ and $c R$ during catalyst operation. The following interpretations are offered:

- The flow rate remains constant at its upper bound during the time the catalyst activity is relatively high. This is because the revenue from 

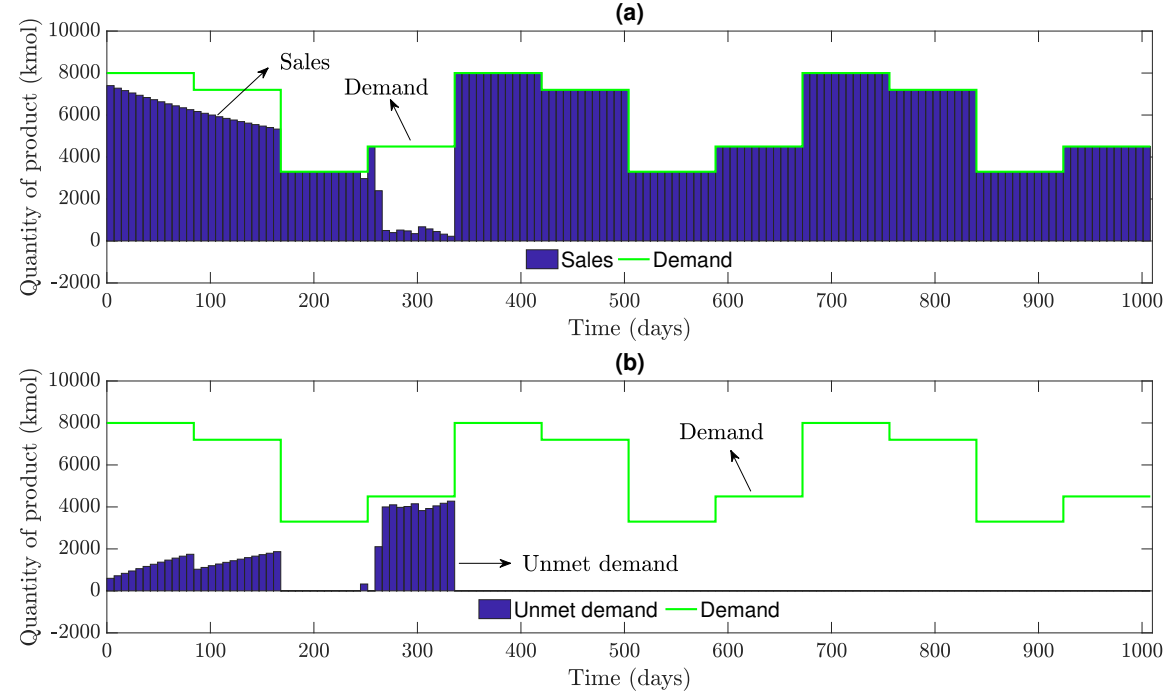

Figure 21: The variation of (a) sales and (b) unmet demand, in comparison to the demand over the time horizon for Case Study C

higher production and lesser unmet demand outweigh the flow costs for this time. Eventually, the catalyst activity falls low enough and causes this balance to shift. At this point, the $f f r$ begins to decrease.

- When $f f r$ begins to decrease, $c R$ begins to decrease from its maximum value. Overall, a large production rate is preferred but at the same time, $f f r$ has to be reduced in order to lower the flow costs. This compromise is attained by decreasing $f f r$ at a rate that minimises the rate of change of $c R$ away from its maximum value and thereby keeps the production rate as large as possible.

- During the operation of the final catalyst, the $f f r$ experiences a sharp drop and exhibits a rate of decrease to result in a production rate that exactly fulfils the demand for the remainder of the time horizon.

(a) 


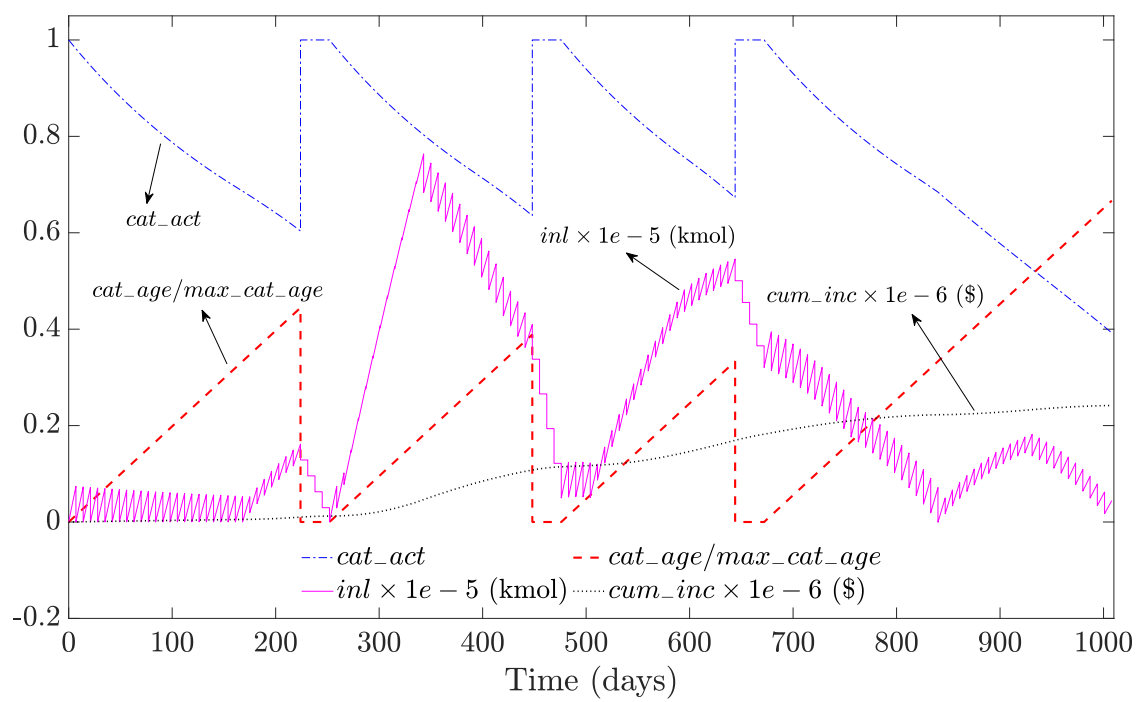

Figure 22: The variation of the catalyst activity, catalyst age, inventory level and cumulative inventory cost over the time horizon for Case Study C

Table 5: Details of the economic aspects for Case Study C

\begin{tabular}{|c|c|c|c|}
\hline \multicolumn{2}{|c|}{ Economic aspect } & Symbol & Value (\$ Millions) \\
\hline \hline \multirow{4}{*}{ Costs } & Gross Revenue from Sales & $G R S$ & 795.192 \\
\hline \hline \multirow{3}{*}{} & Total Inventory Costs & TIC & 0.241 \\
\cline { 2 - 4 } & Total Costs of Catalyst Changeovers & TCCC & 30.999 \\
\cline { 2 - 4 } & Net Penalty for Unmet Demand & NPUD & 93.623 \\
\cline { 2 - 4 } & Total Flow Costs & TFC & 239.836 \\
\hline \hline \multicolumn{2}{|c|}{ Profit } & $-N C$ & 430.493 \\
\hline
\end{tabular}

Figures 21 - 22 and Table 5 are the analogues of Case Study C to Figures 13 - 14 and Table 3 in Case Study A. The profile for the catalyst activity during catalyst operation in Figure 22 follows from equation (11). The explanations for the trends of all variables in Figures 21 and 22 are similar 
to those of their Case Study A analogues. Table 5 reveals that the costs of operation take away about $45.9 \%$ of the revenue generated by the product sales, with the flow costs take up a larger proportion of the total expenses here compared to previous case studies.

\subsubsection{Case Study D: Results and Discussions}

Figures 23 - 26 and Table 6 report the features of the best local optimum among the 50 runs for Case Study D using Implementation II. Here the main reaction is of second order kinetics with respect to the reactant and the catalyst deactivation kinetics is dependent on the product concentration. Such solutions could not be obtained by Implementation I once again, due to problems in integrating the highly nonlinear system of ODEs.

As seen in Figure 23, this solution required two major iterations to force the catalyst changeover controls to take integer values. The suggestion is to use 4 of the 6 available catalysts, with the replacements occurring on the $8^{\text {th }}, 17^{\text {th }}$ and $25^{\text {th }}$ months. Similar to the previous case studies, the timing of these replacements is such that losses are minimised or sufficient inventory is present to meet demand. All other results discussed here are from the solutions of the second major iteration.

The profiles of $f f r$ and $c R$ in Figure 24 are similar to those in Figure 20. Only here, the $f f r$ remains at its maximum value for a longer duration than in Case Study $\mathrm{C}$ because a higher value of $c R$ is needed to compensate for the lower reaction rate.

The explanations for the trends of variables in all other figures are similar their Case Study C analogues. Table 6 reveals that the costs of operation take away about $56.8 \%$ of the revenue generated by the product sales. 


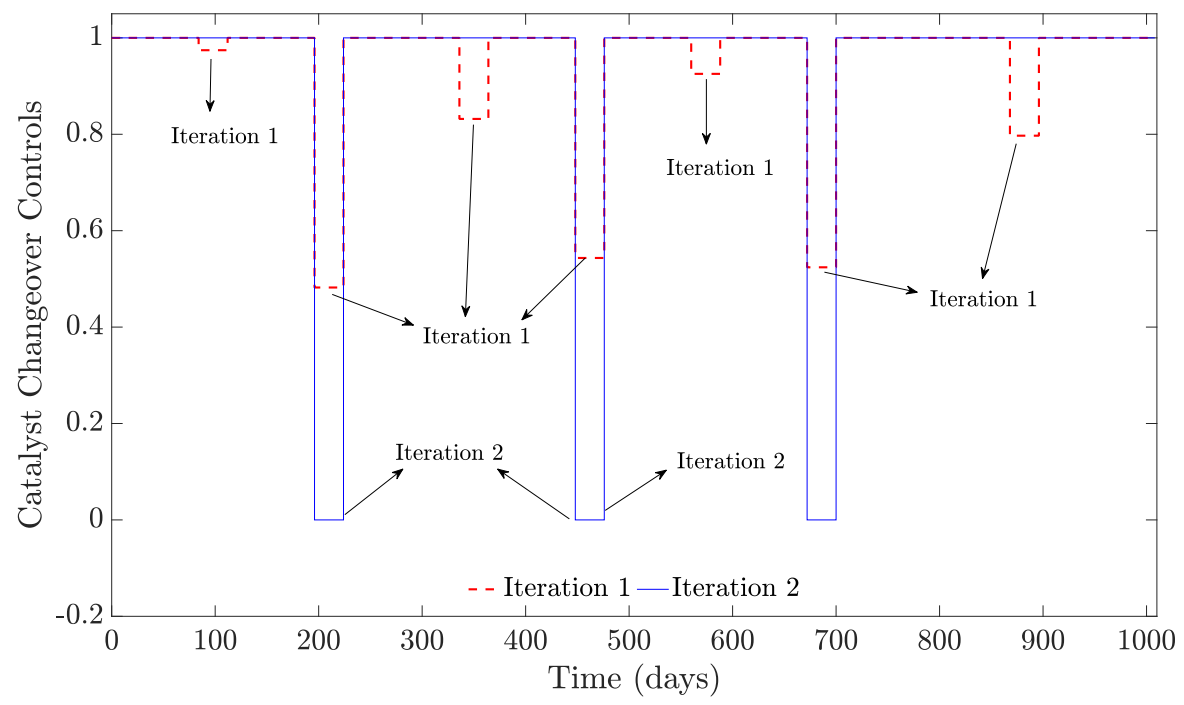

Figure 23: The variation of the catalyst changeover controls over the time horizon for Case Study D

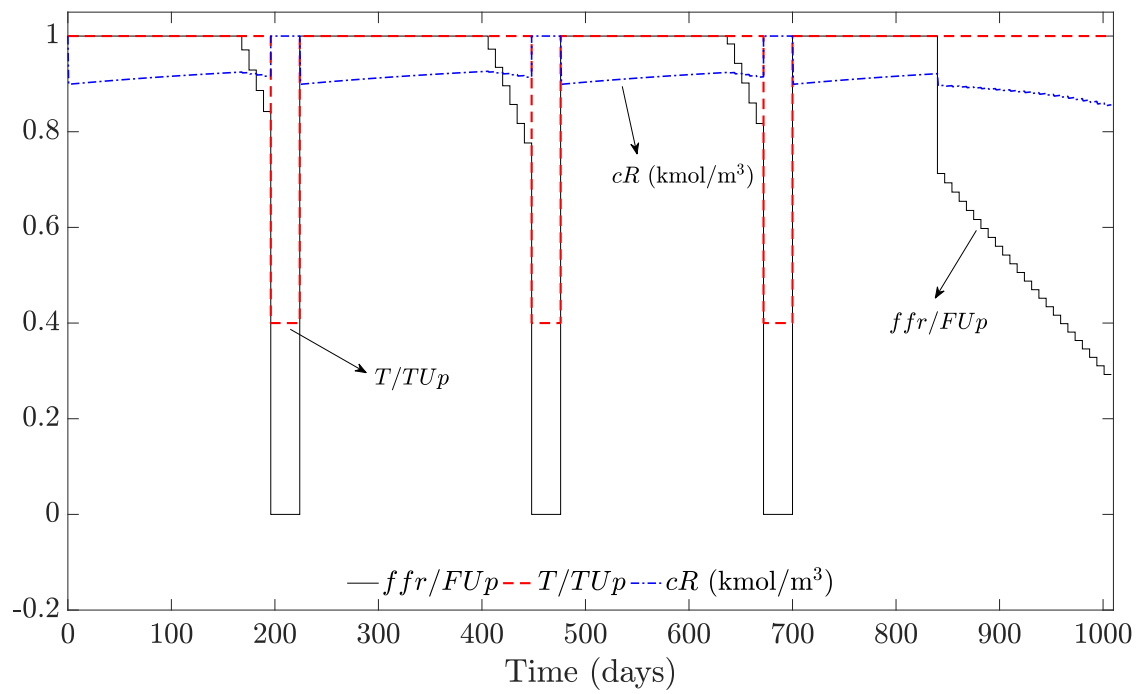

Figure 24: The variation of the feed flow rate, temperature and reactant exit concentration over the time horizon for Case Study D 
(a)

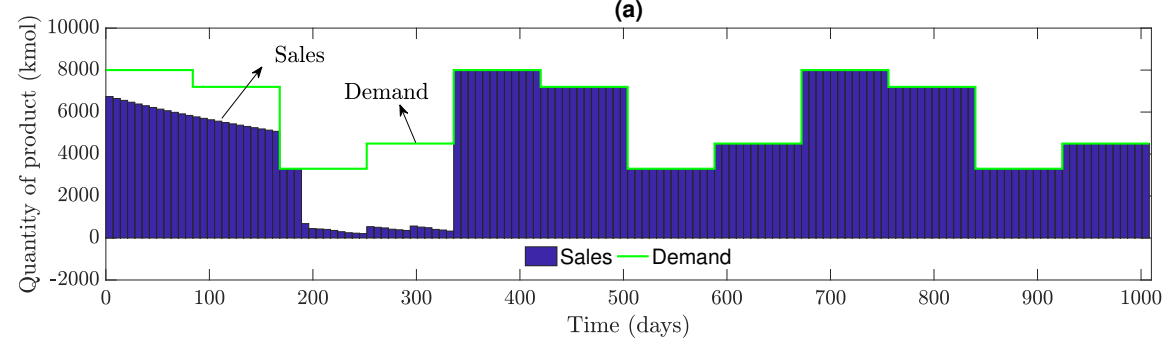

(b)

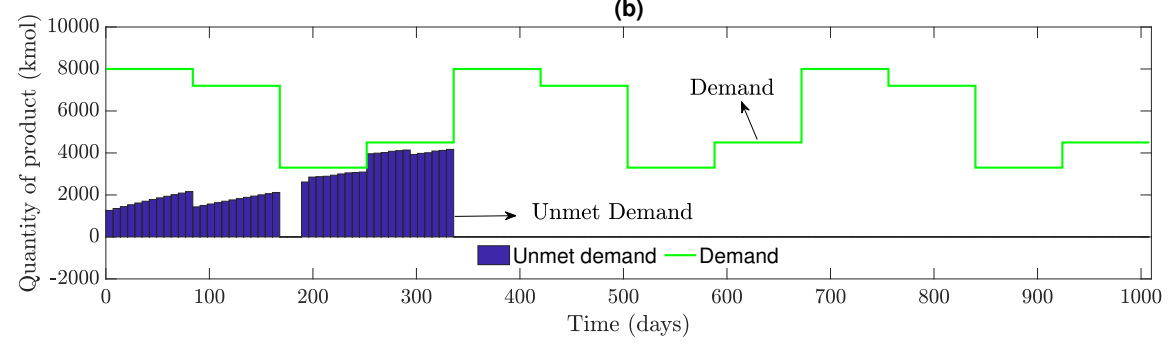

Figure 25: The variation of (a) sales and (b) unmet demand, in comparison to the demand over the time horizon for Case Study D

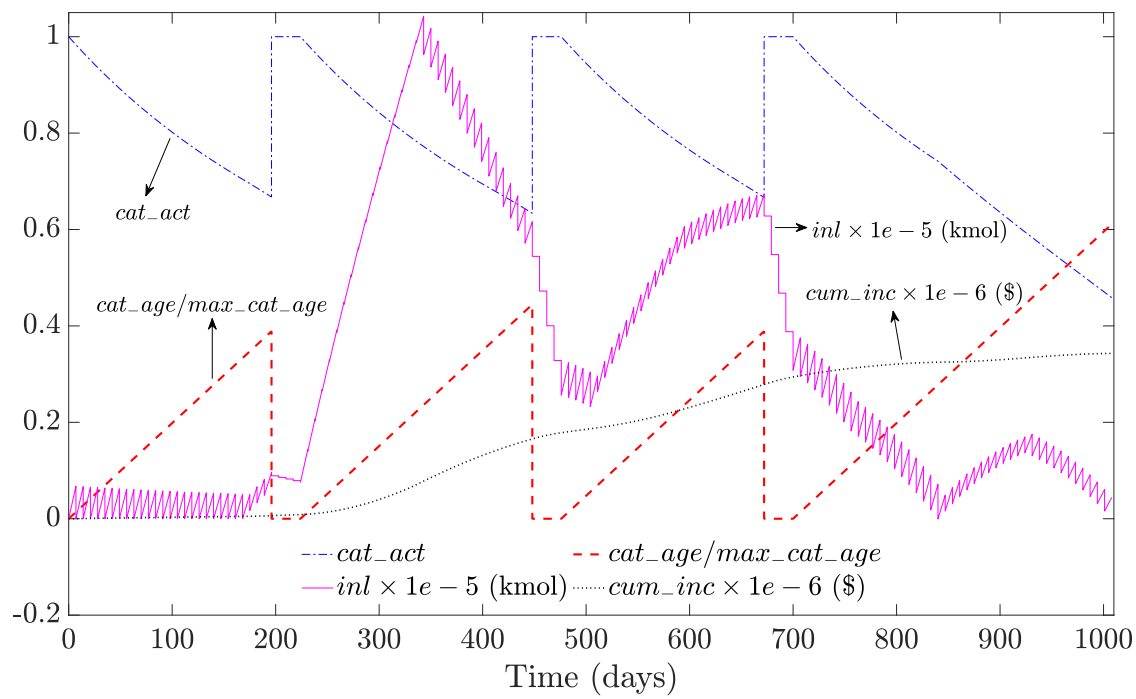

Figure 26: The variation of the catalyst activity, catalyst age, inventory level and cumulative inventory cost over the time horizon for Case Study D 
Table 6: Details of the economic aspects for Case Study D

\begin{tabular}{|c|c|c|c|}
\hline \multicolumn{2}{|c|}{ Economic aspect } & Symbol & Value (\$ Millions) \\
\hline \hline \multirow{3}{*}{ Costs } & Gross Revenue from Sales & $G R S$ & 752.937 \\
\cline { 2 - 4 } & Total Inventory Costs & TIC & 0.343 \\
\cline { 2 - 4 } & Total Costs of Catalyst Changeovers & TCCC & 31.525 \\
\cline { 2 - 4 } & Net Penalty for Unmet Demand & NPUD & 146.441 \\
\cline { 2 - 4 } & Total Flow Costs & TFC & 249.539 \\
\hline \hline \multicolumn{2}{|c|}{ Profit } & $-N C$ & 325.089 \\
\hline
\end{tabular}

999

\section{Conclusions and further discussions}

A novel methodology has been developed to schedule catalyst changeovers and plan production in an industrial process based on the realisation of this problem as a multistage mixed integer optimal control problem. This formulation was applied to four case studies of the process, which differed based on the kinetics of the main reaction or the catalyst deactivation. Due to the non-convex nature of the problem, 50 different starting guesses were used for each case study.

Following from a theoretical analysis of the MSMIOCP formulation, it was expected that the catalyst changeover controls, which appeared affinely in the system equations, should exhibit a bang-bang behaviour in the optimal solution. However, the solution implementation faced complications due to the complex nature of the problem and required using two different implementation methodologies, each of which had their own relative advantages:

1. Implementation I is favourable from a theoretical point of view, as its 
solutions exhibit the bang-bang property for the catalyst changeover controls. However, it has a tendency to converge prematurely or crash due to problems in integration, which are probably due to inadequacies of the MATLAB ODE integrator suite. While a limited set of solutions could be obtained for Case Studies A and B, no solutions could be obtained for case Studies C and D due to these integration problems.

2. Implementation II does not exhibit the bang-bang property for the catalyst changeover controls but is robust and reliable in providing high quality solutions for all case studies. The lack of bang-bang behaviour is most likely an issue of the IPOPT optimiser.

The range of profit values obtained for the successful runs of Implementation I in Case Studies A and B compared well with those in Implementation II, thereby indicating that the answers obtained in these case studies created using invented parameters are indeed optimal.

For each case study, the variation of all control and state variables of the best solution were plotted over the time horizon and the economics of the process was presented in a table. Explanations were provided for the trends of all variables, which were mainly focused on increasing profit while efficiently managing all costs in order to balance the trade-offs involved. A notable result was in Case Study A wherein the policies for reactant exit concentration and temperature of operation correlated well with that of published literature (Szépe and Levenspiel, 1968) at the reactor level. However, the policy for the reactant exit concentration in the solutions of the other case studies was not consistent with the related work (Crowe, 1976) at the reactor level, indicating that that policy may not hold when inventory, sales and demand considerations come into play.

The problem set up considered here is similar to that in Bizet et al. (2005). In order to evaluate the quality of solutions obtained here, a comparison 
between the two works is drawn using the following points:

1. The number of catalyst loads considered in Bizet et al. (2005) was either 2 or 3 . If that number was increased, the number of combinations involved in their solution methodology would increase exponentially and so, obtaining good solutions would require a very large amount of computational effort. On the other hand, the nature of the formulation proposed is such that good solutions can be obtained in a reasonable amount of time even if the number of available catalyst loads is 6 (as considered in this work) or even infinite.

2. In Bizet et al. (2005), the flow rate, temperature and sales are decisions to be taken on a monthly basis, whereas in this work, those controls are optimised on a weekly basis. The smaller problem size enabled by the MSMIOCP approach facilitates producing solutions which are more informative compared to the former. If decisions were taken on a weekly basis in Bizet et al. (2005), the problem size would have increased almost 4-fold, thus accentuating the difficulties in obtaining solutions.

3. The use of integrators to solve the differential equations enables an accurate description of the process dynamics in this work. However, in Bizet et al. (2005) a significant approximation is involved as the differential equations are discretised under a steady state assumption. Thus, the solutions obtained in this work are more reliable.

4. The solution times in Bizet et al. (2005) are in the order of seconds. However, the solution times for the methodology proposed here are in the order of hours, even for a shorter time horizon of 3 years. This is due to the high computational effort spent in solving the differential equations to a high accuracy at each iteration of the optimisation. However, this additional computational effort is not a major issue and is outweighed by the robust, reliable and efficient solutions obtained. 
5. The time horizons considered in Bizet et al. (2005) are 74 months and 9 years. Such long time horizons are unrealistic in present day industries and so, a shorter time horizon of 3 years is considered in this work. However, it is stressed that the methodology proposed here would face no difficulties in producing high quality solutions even for time horizons as long as considered in Bizet et al. (2005).

6. Unlike in this article, no parameters were revealed in Bizet et al. (2005) due to confidentiality reasons and so their results are not reproducible. If such data were available, it would be very interesting to execute the proposed methodology with those parameters and compare the solutions obtained with those of Bizet et al. (2005).

The preceding discussion indicates the high quality of solutions obtained by the proposed methodology in comparison to previous publications. To conclude, the contributions of this paper are highlighted by the following advantages the MSMIOCP approach, employed using Implementation II, offers over existing methodologies:

1. It is robust because solutions can be obtained from any random starting guess, aided by the smaller number of constraints present.

2. It is reliable because solutions can be obtained to a high degree of accuracy using state-of-the-art integrators.

3. It is efficient because the catalyst replacements are scheduled inherently during the optimisation without using combinatorial optimisation methods.

The final points are with regard to the future applications of the proposed methodology. It would be interesting to apply this technique to cases wherein the catalyst deactivation kinetics has a greater dependence on temperature than the main reaction. Another application would be to optimise catalyst 
replacement scheduling and production in a network of reactors, a problem for which numerous MINLP formulations have been developed currently. The consideration of the effect of parametric uncertainties in this problem would also be useful for robust decision making within industry. In addition, while the starting guesses for the decision variables here have been obtained using traditional random number generating functions, it would be interesting to observe the effect of using Latin Hypercube sampling (McKay et al., 1979) or Orthogonal sampling (Tang, 1993), which ensure a better representation of real variability for a random set.

\section{Acknowledgements}

Author S.D. Adloor would like to acknowledge the Cambridge Trust and the Science and Engineering Research Board of India for funding the $\mathrm{PhD}$ studentship for this research.

\section{Appendix A. A Pontryagin analysis of the Multistage Mixed In- teger Optimal Control Problem Formulation}

In this section, a theoretical analysis is performed wherein the Pontryagin Minimum (Maximum) principle is applied to the MSMIOCP formulation developed in Section 2. The performance index in equation (3a) is modified so that Euler-Lagrange multipliers are introduced, as shown in equation (A.1):

$$
\begin{aligned}
\bar{W} & =\sum_{p=2}^{N P}\left\{\left[\phi_{1}^{(p)}\left(x^{(p)}\left(t_{p}\right), z^{(p)}\left(t_{p}\right), v^{(p)}, t_{p}\right)\right]^{T} u^{(p)}+\phi_{2}^{(p)}\left(x^{(p)}\left(t_{p}\right), z^{(p)}\left(t_{p}\right), v^{(p)}, t_{p}\right)\right. \\
& +\left[\nu^{(p)}\right]^{T}\left[E^{(p)}\left(x^{(p-1)}\left(t_{p-1}\right), z^{(p-1)}\left(t_{p-1}\right), v^{(p)}\right) u^{(p)}\right. \\
& \left.+f^{(p)}\left(x^{(p-1)}\left(t_{p-1}\right), z^{(p-1)}\left(t_{p-1}\right), v^{(p)}\right)-x^{(p)}\left(t_{p-1}\right)\right]
\end{aligned}
$$




$$
\begin{aligned}
& +\int_{t_{p-1}}^{t_{p}}\left[\left[L_{1}^{(p)}\left(x^{(p)}(t), z^{(p)}(t), v^{(p)}, t\right)\right]^{T} u^{(p)}+L_{2}^{(p)}\left(x^{(p)}(t), z^{(p)}(t), v^{(p)}, t\right)\right] \mathrm{d} t \\
& +\int_{t_{p-1}}^{t_{p}}\left[\lambda^{(p)}(t)\right]^{T}\left[A^{(p)}\left(x^{(p)}(t), z^{(p)}(t), v^{(p)}, t\right) u^{(p)}\right. \\
& \left.+b^{(p)}\left(x^{(p)}(t), z^{(p)}(t), v^{(p)}, t\right)-\dot{x}^{(p)}(t)\right] \mathrm{d} t \\
& \left.+\int_{t_{p-1}}^{t_{p}}\left[\mu^{(p)}(t)\right]^{T}\left[C^{(p)}\left(x^{(p)}(t), z^{(p)}(t), v^{(p)}, t\right) u^{(p)}+d^{(p)}\left(x^{(p)}(t), z^{(p)}(t), v^{(p)}, t\right)\right] \mathrm{d} t\right\} \\
& +\left[\phi_{1}^{(1)}\left(x^{(1)}\left(t_{1}\right), z^{(1)}\left(t_{1}\right), v^{(1)}, t_{1}\right)\right]^{T} u^{(1)}+\phi_{2}^{(1)}\left(x^{(1)}\left(t_{1}\right), z^{(1)}\left(t_{1}\right), v^{(1)}, t_{1}\right) \\
& +\left[\nu^{(1)}\right]^{T}\left[E^{(1)}\left(v^{(1)}\right) u^{(1)}+f^{(1)}\left(v^{(1)}\right)-x^{(1)}\left(t_{0}\right)\right] \\
& +\int_{t_{0}}^{t_{1}}\left[\left[L_{1}^{(1)}\left(x^{(1)}(t), z^{(1)}(t), v^{(1)}, t\right)\right]^{T} u^{(1)}+L_{2}^{(1)}\left(x^{(1)}(t), z^{(1)}(t), v^{(1)}, t\right)\right] \mathrm{d} t \\
& +\int_{t_{0}}^{t_{1}}\left[\lambda^{(1)}(t)\right]^{T}\left[A^{(1)}\left(x^{(1)}(t), z^{(1)}(t), v^{(1)}, t\right) u^{(1)}\right. \\
& \left.+b^{(1)}\left(x^{(1)}(t), z^{(1)}(t), v^{(1)}, t\right)-\dot{x}^{(1)}(t)\right] \mathrm{d} t \\
& +\int_{t_{0}}^{t_{1}}\left[\mu^{(1)}(t)\right]^{T}\left[C^{(1)}\left(x^{(1)}(t), z^{(1)}(t), v^{(1)}, t\right) u^{(1)}+d^{(1)}\left(x^{(1)}(t), z^{(1)}(t), v^{(1)}, t\right)\right] \mathrm{d} t
\end{aligned}
$$

1120

where $\lambda_{p}, \mu_{p}$ and $\nu_{p}$ are the Euler-Lagrange multipliers for stage $p=1,2, \ldots N P$. Variations on the parameter set of stage $p^{\prime}$ of the form $\delta u\left(p^{\prime}\right)$ are considered, which result in variations in the state values at all times, as shown in equation (A.2). For the sake of convenience, the arguments within the parantheses for each term are neglected. Clearly, the state vector of stage $p$, where $p<p^{\prime}$, will not be influenced. This results in $\delta x^{(p)}(t)=0$ and $\delta y^{(p)}(t)=0$. 


$$
\begin{aligned}
& \delta \bar{W}=\sum_{p=2}^{N P}\left\{\left[\left[u^{(p)}\right]^{T} \frac{\partial \phi_{1}^{(p)}}{\partial x^{(p)}\left(t_{p}\right)}+\frac{\partial \phi_{2}^{(p)}}{\partial x^{(p)}\left(t_{p}\right)}\right] \delta x^{(p)}\left(t_{p}\right)\right. \\
& +\left[\left[u^{(p)}\right]^{T} \frac{\partial \phi_{1}^{(p)}}{\partial z^{(p)}\left(t_{p}\right)}+\frac{\partial \phi_{2}^{(p)}}{\partial z^{(p)}\left(t_{p}\right)}\right] \delta z^{(p)}\left(t_{p}\right) \\
& +\left[\left[u^{(p)}\right]^{T} \frac{\partial \phi_{1}^{(p)}}{\partial v^{(p)}}+\frac{\partial \phi_{2}^{(p)}}{\partial v^{(p)}}\right] \delta v^{(p)}+\left[\phi_{1}^{(p)}\right]^{T} \delta u^{(p)} \\
& +\left[\nu^{(p)}\right]^{T}\left[\left(\frac{\partial E^{(p)}}{\partial x^{(p-1)}\left(t_{p-1}\right)} u^{(p)}+\frac{\partial f^{(p)}}{\partial x^{(p-1)}\left(t_{p-1}\right)}\right) \delta x^{(p-1)}\left(t_{p-1}\right)\right. \\
& +\left(\frac{\partial E^{(p)}}{\partial z^{(p-1)}\left(t_{p-1}\right)} u^{(p)}+\frac{\partial f^{(p)}}{\partial z^{(p-1)}\left(t_{p-1}\right)}\right) \delta z^{(p-1)}\left(t_{p-1}\right) \\
& \left.+\left(\frac{\partial E^{(p)}}{\partial v^{(p)}} u^{(p)}+\frac{\partial f^{(p)}}{\partial v^{(p)}}\right) \delta v^{(p)}+E^{(p)} \delta u^{(p)}-\delta x^{(p)}\left(t_{p-1}\right)\right] \\
& +\int_{t_{p-1}}^{t_{p}}\left[\left(\left[u^{(p)}\right]^{T} \frac{\partial L_{1}^{(p)}}{\partial x^{(p)}}(t)+\frac{\partial L_{2}^{(p)}}{\partial x^{(p)}}(t)\right) \delta x^{(p)}(t)\right. \\
& +\left(\left[u^{(p)}\right]^{T} \frac{\partial L_{1}^{(p)}}{\partial z^{(p)}}(t)+\frac{\partial L_{2}^{(p)}}{\partial z^{(p)}}(t)\right) \delta z^{(p)}(t) \\
& \left.+\left(\left[u^{(p)}\right]^{T} \frac{\partial L_{1}^{(p)}(t)}{\partial v^{(p)}}+\frac{\partial L_{2}^{(p)}(t)}{\partial v^{(p)}}\right) \delta v^{(p)}+\left[L_{1}^{(p)}\right]^{T} \delta u^{(p)}\right] \mathrm{d} t \\
& +\int_{t_{p-1}}^{t_{p}}\left[\lambda^{(p)}(t)\right]^{T}\left[\left(\frac{\partial A^{(p)}}{\partial x^{(p)}}(t) u^{(p)}+\frac{\partial b^{(p)}}{\partial x^{(p)}}(t)\right) \delta x^{(p)}(t)\right. \\
& +\left(\frac{\partial A^{(p)}}{\partial z^{(p)}}(t) u^{(p)}+\frac{\partial b^{(p)}}{\partial z^{(p)}}(t)\right) \delta z^{(p)}(t)+\left(\frac{\partial A^{(p)}(t)}{\partial v^{(p)}} u^{(p)}+\frac{\partial b^{(p)}(t)}{\partial v^{(p)}}\right) \delta v^{(p)} \\
& \left.+A^{(p)} \delta u^{(p)}-\delta \dot{x}^{(p)}(t)\right] \mathrm{d} t
\end{aligned}
$$




$$
\begin{aligned}
& +\int_{t_{p-1}}^{t_{p}}\left[\mu^{(p)}(t)\right]^{T}\left[\left(\frac{\partial C^{(p)}}{\partial x^{(p)}}(t) u^{(p)}+\frac{\partial d^{(p)}}{\partial x^{(p)}}(t)\right) \delta x^{(p)}(t)\right. \\
& +\left(\frac{\partial C^{(p)}}{\partial z^{(p)}}(t) u^{(p)}+\frac{\partial d^{(p)}}{\partial z^{(p)}}(t)\right) \delta z^{(p)}(t) \\
& \left.\left.+\left(\frac{\partial C^{(p)}(t)}{\partial v^{(p)}} u^{(p)}+\frac{\partial d^{(p)}(t)}{\partial v^{(p)}}\right) \delta v^{(p)}+C^{(p)} \delta u^{(p)}\right] \mathrm{d} t\right\} \\
& +\left[\left(\left[u^{(1)}\right]^{T} \frac{\partial \phi_{1}^{(1)}}{\partial x^{(1)}\left(t_{1}\right)}+\frac{\partial \phi_{2}^{(1)}}{\partial x^{(1)}\left(t_{1}\right)}\right) \delta x^{(1)}\left(t_{1}\right)\right. \\
& +\left(\left[u^{(1)}\right]^{T} \frac{\partial \phi_{1}^{(1)}}{\partial z^{(1)}\left(t_{1}\right)}+\frac{\partial \phi_{2}^{(1)}}{\partial z^{(1)}\left(t_{1}\right)}\right) \delta z^{(1)}\left(t_{1}\right) \\
& \left.+\left(\left[u^{(1)}\right]^{T} \frac{\partial \phi_{1}^{(1)}}{\partial v^{(1)}}+\frac{\partial \phi_{2}^{(1)}}{\partial v^{(1)}}\right) \delta v^{(1)}+\left[\phi_{1}^{(1)}\right]^{T} \delta u^{(1)}\right] \\
& +\left[\nu^{(1)}\right]^{T}\left[\left(\frac{\partial E^{(1)}}{\partial v^{(1)}} u^{(1)}+\frac{\partial f^{(1)}}{\partial v^{(1)}}\right) \delta v^{(1)}+E^{(1)} \delta u^{(1)}-\delta x^{(1)}\left(t_{0}\right)\right] \\
& +\int_{t_{0}}^{t_{1}}\left[\left(\left[u^{(1)}\right]^{T} \frac{\partial L_{1}^{(1)}}{\partial x^{(1)}}(t)+\frac{\partial L_{2}^{(1)}}{\partial x^{(1)}}(t)\right) \delta x^{(1)}(t)\right. \\
& +\left(\left[u^{(1)}\right]^{T} \frac{\partial L_{1}^{(1)}}{\partial z^{(1)}}(t)+\frac{\partial L_{2}^{(1)}}{\partial z^{(1)}}(t)\right) \delta z^{(1)}(t) \\
& \left.+\left(\left[u^{(1)}\right]^{T} \frac{\partial L_{1}^{(1)}(t)}{\partial v^{(1)}}+\frac{\partial L_{2}^{(1)}(t)}{\partial v^{(1)}}\right) \delta v^{(1)}+\left[L_{1}^{(1)}\right]^{T} \delta u^{(1)}\right] \mathrm{d} t \\
& +\int_{t_{0}}^{t_{1}}\left[\lambda^{(1)}(t)\right]^{T}\left[\left(\frac{\partial A^{(1)}}{\partial x^{(1)}}(t) u^{(1)}+\frac{\partial b^{(1)}}{\partial x^{(1)}}(t)\right) \delta x^{(1)}(t)\right. \\
& +\left(\frac{\partial A^{(1)}}{\partial z^{(1)}}(t) u^{(1)}+\frac{\partial b^{(1)}}{\partial z^{(1)}}(t)\right) \delta z^{(1)}(t) \\
& \left.+\left(\frac{\partial A^{(1)}(t)}{\partial v^{(1)}} u^{(1)}+\frac{\partial b^{(1)}(t)}{\partial v^{(1)}}\right) \delta v^{(1)}+A^{(1)} \delta u^{(1)}-\delta \dot{x}^{(1)}(t)\right] \mathrm{d} t \\
& +\int_{t_{0}}^{t_{1}}\left[\mu^{(1)}(t)\right]^{T}\left[\left(\frac{\partial C^{(1)}}{\partial x^{(1)}}(t) u^{(1)}+\frac{\partial d^{(1)}}{\partial x^{(1)}}(t)\right) \delta x^{(1)}(t)\right.
\end{aligned}
$$




$$
\begin{aligned}
& +\left(\frac{\partial C^{(1)}}{\partial z^{(1)}}(t) u^{(1)}+\frac{\partial d^{(1)}}{\partial z^{(1)}}(t)\right) \delta z^{(1)}(t) \\
& \left.+\left(\frac{\partial C^{(1)}(t)}{\partial v^{(1)}} u^{(1)}+\frac{\partial d^{(1)}(t)}{\partial v^{(1)}}\right) \delta v^{(1)}+C^{(1)} \delta u^{(1)}\right] \mathrm{d} t
\end{aligned}
$$

$$
\begin{aligned}
\delta \bar{W}= & \sum_{p=2}^{N P}\left\{\left[\left[u^{(p)}\right]^{T} \frac{\partial \phi_{1}^{(p)}}{\partial x^{(p)}\left(t_{p}\right)}+\frac{\partial \phi_{2}^{(p)}}{\partial x^{(p)}\left(t_{p}\right)}\right] \delta x^{(p)}\left(t_{p}\right)\right. \\
& +\left[\left[u^{(p)}\right]^{T} \frac{\partial \phi_{1}^{(p)}}{\partial z^{(p)}\left(t_{p}\right)}+\frac{\partial \phi_{2}^{(p)}}{\partial z^{(p)}\left(t_{p}\right)}\right] \delta z^{(p)}\left(t_{p}\right) \\
& +\left[\left[u^{(p)}\right]^{T} \frac{\partial \phi_{1}^{(p)}}{\partial v^{(p)}}+\frac{\partial \phi_{2}^{(p)}}{\partial v^{(p)}}\right] \delta v^{(p)}+\left[\phi_{1}^{(p)}\right]^{T} \delta u^{(p)} \\
& +\left[\nu^{(p)}\right]^{T}\left[\left(\frac{\partial E^{(p)}}{\partial x^{(p-1)}\left(t_{p-1}\right)} u^{(p)}+\frac{\partial f^{(p)}}{\partial x^{(p-1)}\left(t_{p-1}\right)}\right) \delta x^{(p-1)}\left(t_{p-1}\right)\right. \\
& +\left(\frac{\partial E^{(p)}}{\partial z^{(p-1)}\left(t_{p-1}\right)} u^{(p)}+\frac{\partial f^{(p)}}{\partial z^{(p-1)}\left(t_{p-1}\right)}\right) \delta z^{(p-1)}\left(t_{p-1}\right) \\
& \left.+\left(\frac{\partial E^{(p)}}{\partial v^{(p)}} u^{(p)}+\frac{\partial f^{(p)}}{\partial v^{(p)}}\right) \delta v^{(p)}+E^{(p)} \delta u^{(p)}-\delta x^{(p)}\left(t_{p-1}\right)\right] \\
& +\int_{t_{p-1}}^{t_{p}}\left[\left(\left[u^{(p)}\right]^{T} \frac{\partial L_{1}^{(p)}}{\partial x^{(p)}}(t)+\frac{\partial L_{2}^{(p)}}{\partial x^{(p)}}(t)\right) \delta x^{(p)}(t)\right. \\
& +\left(\left[u^{(p)}\right]^{T} \frac{\partial L_{1}^{(p)}}{\partial z^{(p)}}(t)+\frac{\partial L_{2}^{(p)}}{\partial z^{(p)}}(t)\right) \delta z^{(p)}(t)
\end{aligned}
$$




$$
\begin{aligned}
& \left.+\left(\left[u^{(p)}\right]^{T} \frac{\partial L_{1}^{(p)}(t)}{\partial v^{(p)}}+\frac{\partial L_{2}^{(p)}(t)}{\partial v^{(p)}}\right) \delta v^{(p)}+\left[L_{1}^{(p)}\right]^{T} \delta u^{(p)}\right] \mathrm{d} t \\
& +\int_{t_{p-1}}^{t_{p}}\left[\lambda^{(p)}(t)\right]^{T}\left[\left(\frac{\partial A^{(p)}}{\partial x^{(p)}}(t) u^{(p)}+\frac{\partial b^{(p)}}{\partial x^{(p)}}(t)\right) \delta x^{(p)}(t)\right. \\
& +\left(\frac{\partial A^{(p)}}{\partial z^{(p)}}(t) u^{(p)}+\frac{\partial b^{(p)}}{\partial z^{(p)}}(t)\right) \delta z^{(p)}(t) \\
& \left.+\left(\frac{\partial A^{(p)}(t)}{\partial v^{(p)}} u^{(p)}+\frac{\partial b^{(p)}(t)}{\partial v^{(p)}}\right) \delta v^{(p)}+A^{(p)} \delta u^{(p)}\right] \mathrm{d} t \\
& +\int_{t_{p-1}}^{t_{p}}\left[\left[\dot{\lambda}^{(p)}(t)\right]^{T} \delta x^{(p)}(t)\right] \mathrm{d} t+\left[\lambda^{(p)}\left(t_{p-1}\right)\right]^{T} \delta x^{(p)}\left(t_{p-1}\right)-\left[\lambda^{(p)}\left(t_{p}\right)\right]^{T} \delta x^{(p)}\left(t_{p}\right) \\
& +\int_{t_{p-1}}^{t_{p}}\left[\mu^{(p)}(t)\right]^{T}\left[\left(\frac{\partial C^{(p)}}{\partial x^{(p)}}(t) u^{(p)}+\frac{\partial d^{(p)}}{\partial x^{(p)}}(t)\right) \delta x^{(p)}(t)\right. \\
& +\left(\frac{\partial C^{(p)}}{\partial z^{(p)}}(t) u^{(p)}+\frac{\partial d^{(p)}}{\partial z^{(p)}}(t)\right) \delta z^{(p)}(t) \\
& \left.\left.+\left(\frac{\partial C^{(p)}(t)}{\partial v^{(p)}} u^{(p)}+\frac{\partial d^{(p)}(t)}{\partial v^{(p)}}\right) \delta v^{(p)}+C^{(p)} \delta u^{(p)}\right] \mathrm{d} t\right\} \\
& +\left[\left(\left[u^{(1)}\right]^{T} \frac{\partial \phi_{1}^{(1)}}{\partial x^{(1)}\left(t_{1}\right)}+\frac{\partial \phi_{2}^{(1)}}{\partial x^{(1)}\left(t_{1}\right)}\right) \delta x^{(1)}\left(t_{1}\right)\right. \\
& +\left(\left[u^{(1)}\right]^{T} \frac{\partial \phi_{1}^{(1)}}{\partial z^{(1)}\left(t_{1}\right)}+\frac{\partial \phi_{2}^{(1)}}{\partial z^{(1)}\left(t_{1}\right)}\right) \delta z^{(1)}\left(t_{1}\right) \\
& \left.+\left(\left[u^{(1)}\right]^{T} \frac{\partial \phi_{1}^{(1)}}{\partial v^{(1)}}+\frac{\partial \phi_{2}^{(1)}}{\partial v^{(1)}}\right) \delta v^{(1)}+\left[\phi_{1}^{(1)}\right]^{T} \delta u^{(1)}\right] \\
& +\left[\nu^{(1)}\right]^{T}\left[\left(\frac{\partial E^{(1)}}{\partial v^{(1)}} u^{(1)}+\frac{\partial f^{(1)}}{\partial v^{(1)}}\right) \delta v^{(1)}+E^{(1)} \delta u^{(1)}-\delta x^{(1)}\left(t_{0}\right)\right] \\
& +\int_{t_{0}}^{t_{1}}\left[\left(\left[u^{(1)}\right]^{T} \frac{\partial L_{1}^{(1)}}{\partial x^{(1)}}(t)+\frac{\partial L_{2}^{(1)}}{\partial x^{(1)}}(t)\right) \delta x^{(1)}(t)\right. \\
& +\left(\left[u^{(1)}\right]^{T} \frac{\partial L_{1}^{(1)}}{\partial z^{(1)}}(t)+\frac{\partial L_{2}^{(1)}}{\partial z^{(1)}}(t)\right) \delta z^{(1)}(t)
\end{aligned}
$$




$$
\begin{aligned}
& \left.+\left(\left[u^{(1)}\right]^{T} \frac{\partial L_{1}^{(1)}(t)}{\partial v^{(1)}}+\frac{\partial L_{2}^{(1)}(t)}{\partial v^{(1)}}\right) \delta v^{(1)}+\left[L_{1}^{(1)}\right]^{T} \delta u^{(1)}\right] \mathrm{d} t \\
& +\int_{t_{0}}^{t_{1}}\left[\lambda^{(1)}(t)\right]^{T}\left[\left(\frac{\partial A^{(1)}}{\partial x^{(1)}}(t) u^{(1)}+\frac{\partial b^{(1)}}{\partial x^{(1)}}(t)\right) \delta x^{(1)}(t)\right. \\
& +\left(\frac{\partial A^{(1)}}{\partial z^{(1)}}(t) u^{(1)}+\frac{\partial b^{(1)}}{\partial z^{(1)}}(t)\right) \delta z^{(1)}(t) \\
& \left.+\left(\frac{\partial A^{(1)}(t)}{\partial v^{(1)}} u^{(1)}+\frac{\partial b^{(1)}(t)}{\partial v^{(1)}}\right) \delta v^{(1)}+A^{(1)} \delta u^{(1)}\right] \mathrm{d} t \\
& +\int_{t_{0}}^{t_{1}}\left[\left[\dot{\lambda}^{(1)}(t)\right]^{T} \delta x^{(1)}(t)\right] \mathrm{d} t+\left[\lambda^{(1)}\left(t_{0}\right)\right]^{T} \delta x^{(1)}\left(t_{0}\right)-\left[\lambda^{(1)}\left(t_{1}\right)\right]^{T} \delta x^{(1)}\left(t_{1}\right) \\
& +\int_{t_{0}}^{t_{1}}\left[\mu^{(1)}(t)\right]^{T}\left[\left(\frac{\partial C^{(1)}}{\partial x^{(1)}}(t) u^{(1)}+\frac{\partial d^{(1)}}{\partial x^{(1)}}(t)\right) \delta x^{(1)}(t)\right. \\
& +\left(\frac{\partial C^{(1)}}{\partial z^{(1)}}(t) u^{(1)}+\frac{\partial d^{(1)}}{\partial z^{(1)}}(t)\right) \delta z^{(1)}(t) \\
& \left.+\left(\frac{\partial C^{(1)}(t)}{\partial v^{(1)}} u^{(1)}+\frac{\partial d^{(1)}(t)}{\partial v^{(1)}}\right) \delta v^{(1)}+C^{(1)} \delta u^{(1)}\right] \mathrm{d} t
\end{aligned}
$$

For a stationary point, infinitesimal variations in the right hand side should yield no change to the performance index, i.e. $\delta \bar{W}=0$, and hence related terms must be chosen so that they always guarantee this. This leads to the following set of Euler-Lagrange equations and the Pontryagin Minimum (Maximum) principle (Pontryagin et al., 1962).

To cancel the $\delta x^{(1)}$ and $\delta x^{(1)}\left(t_{1}\right)$ terms, the differential equations and final time stage conditions, as shown in equations (A.4a) - (A.5), must hold, respectively: 


$$
\begin{aligned}
\dot{\lambda}^{(1)}(t) & =-\left[\frac{\partial A^{(1)}}{\partial x^{(1)}}(t) u^{(1)}+\frac{\partial b^{(1)}}{\partial x^{(1)}}(t)\right]^{T}\left[\lambda^{(1)}(t)\right]-\left[\frac{\partial C^{(1)}}{\partial x^{(1)}}(t) u^{(1)}+\frac{\partial d^{(1)}}{\partial x^{(1)}}(t)\right]^{T}\left[\mu^{(1)}(t)\right] \\
& -\left[\left[u^{(1)}\right]^{T} \frac{\partial L_{1}^{(1)}}{\partial x^{(1)}}(t)+\frac{\partial L_{2}^{(1)}}{\partial x^{(1)}}(t)\right]^{T}
\end{aligned}
$$

1137

$$
t_{0} \leq t \leq t_{1}
$$

$$
\lambda^{(1)}\left(t_{1}\right)=\left[\left[u^{(1)}\right]^{T} \frac{\partial \phi_{1}^{(1)}}{\partial x^{(1)}\left(t_{1}\right)}+\frac{\partial \phi_{2}^{(1)}}{\partial x^{(1)}\left(t_{1}\right)}\right]^{T}+\left[\frac{\partial E^{(2)}}{\partial x^{(1)}\left(t_{1}\right)} u^{(2)}+\frac{\partial f^{(2)}}{\partial x^{(1)}\left(t_{1}\right)}\right]^{T} \nu^{(2)}
$$

1138 To cancel the $\delta z^{(1)}$ and $\delta z^{(1)}\left(t_{1}\right)$ terms, the algebraic equations and final 1139 time stage conditions, as shown in equations (A.6a) - (A.7), must hold, 1140 respectively:

$$
\begin{aligned}
{\left[\frac{\partial A^{(1)}}{\partial z^{(1)}}(t) u^{(1)}+\frac{\partial b^{(1)}}{\partial z^{(1)}}(t)\right]^{T}\left[\lambda^{(1)}(t)\right]+} & {\left[\frac{\partial C^{(1)}}{\partial z^{(1)}}(t) u^{(1)}+\frac{\partial d^{(1)}}{\partial z^{(1)}}(t)\right]^{T}\left[\mu^{(1)}(t)\right] } \\
+ & {\left[\left[u^{(1)}\right]^{T} \frac{\partial L_{1}^{(1)}}{\partial z^{(1)}}(t)+\frac{\partial L_{2}^{(1)}}{\partial z^{(1)}}(t)\right]^{T}=0 }
\end{aligned}
$$

1141

1142

$$
\left[\left[u^{(1)}\right]^{T} \frac{\partial \phi_{1}^{(1)}}{\partial z^{(1)}\left(t_{1}\right)}+\frac{\partial \phi_{2}^{(1)}}{\partial z^{(1)}\left(t_{1}\right)}\right]^{T}+\left[\frac{\partial E^{(2)}}{\partial z^{(1)}\left(t_{1}\right)} u^{(2)}+\frac{\partial f^{(2)}}{\partial z^{(1)}\left(t_{1}\right)}\right]^{T} \nu^{(2)}=0
$$

1143 1144 1145

The $\delta x^{(p)}(t), \delta x^{(p)}\left(t_{p}\right)$ and $\delta x^{(p)}\left(t_{p-1}\right)$ terms are cancelled through the condition that the following differential equations and final time stage conditions (equations (A.8a) - (A.10)) hold: 


$$
\begin{aligned}
\dot{\lambda}^{(p)}(t) & =-\left[\frac{\partial A^{(p)}}{\partial x^{(p)}}(t) u^{(p)}+\frac{\partial b^{(p)}}{\partial x^{(p)}}(t)\right]^{T}\left[\lambda^{(p)}(t)\right]-\left[\frac{\partial C^{(p)}}{\partial x^{(p)}}(t) u^{(p)}+\frac{\partial d^{(p)}}{\partial x^{(p)}}(t)\right]^{T}\left[\mu^{(p)}(t)\right] \\
& -\left[\left[u^{(p)}\right]^{T} \frac{\partial L_{1}^{(p)}}{\partial x^{(p)}}(t)+\frac{\partial L_{2}^{(p)}}{\partial x^{(p)}}(t)\right]^{T}
\end{aligned}
$$

1146

$$
\begin{gathered}
t_{p-1} \leq t \leq t_{p} \quad p=2,3, \ldots, N P \\
\lambda^{(p)}\left(t_{p}\right)=\left[\left[u^{(p)}\right]^{T} \frac{\partial \phi_{1}^{(p)}}{\partial x^{(p)}\left(t_{p}\right)}+\frac{\partial \phi_{2}^{(p)}}{\partial x^{(p)}\left(t_{p}\right)}\right]^{T}+\left[\frac{\partial E^{(p+1)}}{\partial x^{(p)}\left(t_{p}\right)} u^{(p+1)}+\frac{\partial f^{(p+1)}}{\partial x^{(p)}\left(t_{p}\right)}\right]^{T} \nu^{(p+1)} \\
p=2,3, \ldots, N P-1 \\
\lambda^{(p)}\left(t_{p}\right)=\left[\left[u^{(p)}\right]^{T} \frac{\partial \phi_{1}^{(p)}}{\partial x^{(p)}\left(t_{p}\right)}+\frac{\partial \phi_{2}^{(p)}}{\partial x^{(p)}\left(t_{p}\right)}\right]^{T} \\
p=N P \\
\nu^{(p)}=\lambda^{(p)}\left(t_{p-1}\right) \\
p=2,3, \ldots, N \mathrm{~b})
\end{gathered}
$$

1147 Algebraic equations and final stage conditions, equations (A.11a) - (A.12b) ${ }_{1148}$ must hold in order to cancel the $\delta z^{(p)}$ and $\delta z^{(p)}\left(t_{p}\right)$ terms.

$$
\begin{aligned}
& {\left[\frac{\partial A^{(p)}}{\partial z^{(p)}}(t) u^{(p)}+\frac{\partial b^{(p)}}{\partial z^{(p)}}(t)\right]^{T}\left[\lambda^{(p)}(t)\right]+} {\left[\frac{\partial C^{(p)}}{\partial z^{(p)}}(t) u^{(p)}+\frac{\partial d^{(p)}}{\partial z^{(p)}}(t)\right]^{T}\left[\mu^{(p)}(t)\right] } \\
&+\left[\left[u^{(p)}\right]^{T} \frac{\partial L_{1}^{(p)}}{\partial z^{(p)}}(t)+\frac{\partial L_{2}^{(p)}}{\partial z^{(p)}}(t)\right]^{T}=0
\end{aligned}
$$




$$
\begin{gathered}
t_{p-1} \leq t \leq t_{p} \quad p=2,3, \ldots, N P \\
{\left[\left[u^{(p)}\right]^{T} \frac{\partial \phi_{1}^{(p)}}{\partial z^{(p)}\left(t_{p}\right)}+\frac{\partial \phi_{2}^{(p)}}{\partial z^{(p)}\left(t_{p}\right)}\right]^{T}+\left[\frac{\partial E^{(p+1)}}{\partial z^{(p)}\left(t_{p}\right)} u^{(p+1)}+\frac{\partial f^{(p+1)}}{\partial z^{(p)}\left(t_{p}\right)}\right]^{T} \nu^{(p+1)}=0} \\
p=2,3, \ldots, N P-1 \\
{\left[\left[u^{(p)}\right]^{T} \frac{\partial \phi_{1}^{(p)}}{\partial z^{(p)}\left(t_{p}\right)}+\frac{\partial \phi_{2}^{(p)}}{\partial z^{(p)}\left(t_{p}\right)}\right]^{T}=0} \\
p=N P \\
\quad \text { (A.12a) } \\
\text { (A.12b) }
\end{gathered}
$$

1149 As per the Pontryagin Minimum (Maximum) Principle, the decision vari1150 ables of the problem should be chosen to minimise the Hamiltonian. The 1151 Hamiltonian gradient conditions, taken from the coefficients of $\delta v^{(p)}$ and $\delta u^{(p)}$, 1152 are given by equations (A.13a) - (A.14b).

$$
\begin{aligned}
\nabla_{v^{(p)}} H^{(p)}= & {\left[\left[u^{(p)}\right]^{T} \frac{\partial \phi_{1}^{(p)}}{\partial v^{(p)}}+\frac{\partial \phi_{2}^{(p)}}{\partial v^{(p)}}\right]^{T}+\left[\frac{\partial E^{(p)}}{\partial v^{(p)}} u^{(p)}+\frac{\partial f^{(p)}}{\partial v^{(p)}}\right]^{T} \nu^{(p)} } \\
& +\int_{t_{p-1}}^{t_{p}}\left[\left[\left[u^{(p)}\right]^{T} \frac{\partial L_{1}^{(p)}(t)}{\partial v^{(p)}}+\frac{\partial L_{2}^{(p)}(t)}{\partial v^{(p)}}\right]^{T}\right. \\
& +\left[\frac{\partial A^{(p)}(t)}{\partial v^{(p)}} u^{(p)}+\frac{\partial b^{(p)}(t)}{\partial v^{(p)}}\right]^{T} \lambda^{(p)}(t) \\
& \left.+\left[\frac{\partial C^{(p)}(t)}{\partial v^{(p)}} u^{(p)}+\frac{\partial d^{(p)}(t)}{\partial v^{(p)}}\right]^{T} \mu^{(p)}(t)\right] \mathrm{d} t \\
& =0
\end{aligned}
$$


1153

$$
\begin{aligned}
& t_{p-1} \leq t \leq t_{p} \quad p=1,2, \ldots, N P \\
\nabla_{u^{(p)}} H^{(p)}= & \phi_{1}^{(p)}+\left[E^{(p)}\right]^{T} \nu^{(p)} \\
& +\int_{t_{p-1}}^{t_{p}}\left[L_{1}^{(p)}+\left[A^{(p)}\right]^{T} \lambda^{(p)}(t)+\left[C^{(p)}\right]^{T} \mu^{(p)}(t)\right] \mathrm{d} t \\
& =0
\end{aligned}
$$

1154

$$
t_{p-1} \leq t \leq t_{p} \quad p=1,2, \ldots, N P
$$

\section{Appendix B. Tables}

Table B.7: List of parameters

\begin{tabular}{|c|c|}
\hline Parameter Symbol & Value \\
\hline \hline$A_{R}$ & $885(1 /$ day $)$ \\
\hline base_cof & $\$ 210 /$ week \\
\hline base_crc & $\$ 10^{7}$ \\
\hline base_icf & $\$ 0.01 /(\mathrm{kmol}$ day $)$ \\
\hline base_pen & $\$ 1250 / \mathrm{kmol}$ \\
\hline base_psp & $\$ 1000 / \mathrm{kmol}$ \\
\hline \multirow{2}{*}{$C R 0$} & $1 \mathrm{kmol} / \mathrm{m}^{3}$ \\
\hline \multirow{2}{*}{ demand } & 1st quarter of year: $8000 \mathrm{kmol} /$ week \\
\cline { 2 - 3 } & 2nd quarter of year: $7200 \mathrm{kmol} /$ week \\
\cline { 2 - 3 } & 3rd quarter of year: $3300 \mathrm{kmol} /$ week \\
\cline { 2 - 3 } & 4th quarter of year: $4500 \mathrm{kmol} /$ week \\
\hline \multirow{2}{*}{$E_{\text {act }}$} & $30,000 \mathrm{~J} / \mathrm{gmol}$ \\
\hline
\end{tabular}


Table B.7: List of parameters

\begin{tabular}{|c|c|}
\hline Parameter Symbol & Value \\
\hline \hline FUp & $9600 \mathrm{~m}^{3} /$ day \\
\hline inflation & $5 \%$ \\
\hline \multirow{2}{*}{$K_{d}$} & Case Study A: $0.0024(1 /$ day $)$ \\
\cline { 2 - 2 } & Case Study B: $0.0024\left(1 /\left(\right.\right.$ day $\left.\left.\cdot \mathrm{kmol} / \mathrm{m}^{3}\right)\right)$ \\
\cline { 2 - 2 } & Case Studies C, D: $0.024\left(1 /\left(\right.\right.$ day $\left.\left.\cdot \mathrm{kmol} / \mathrm{m}^{3}\right)\right)$ \\
\hline max_cat_age & 504 days $(=1.5$ years $)$ \\
\hline \multirow{2}{*}{$N$} & 56 months $(=3$ years $)$ \\
\hline$R_{g}$ & $8.314 \mathrm{~J} /(\mathrm{gmol} . \mathrm{K})$ \\
\hline start_cat_act & 1 \\
\hline$T L o$ & $400 \mathrm{~K}$ \\
\hline$T U p$ & $1000 \mathrm{~K}$ \\
\hline VR & $50 \mathrm{~m}^{3}$ \\
\hline
\end{tabular}


Table B.8: Problem size specifications, applicable for each case study

\begin{tabular}{|c|c|c|}
\hline \multicolumn{2}{|c|}{ Property } & Size \\
\hline \hline Ordinary & Differential Equations & 720 \\
\hline \hline \multirow{4}{*}{ Decision variables } & Catalyst changeover actions & 36 \\
\cline { 2 - 3 } & Feed flow rate & 144 \\
\cline { 2 - 3 } & Sales & 144 \\
\cline { 2 - 3 } & Temperature & 144 \\
\cline { 2 - 3 } & Total & 468 \\
\hline \hline \multirow{5}{*}{ Constraints } & Constraints (29) & 72 \\
\cline { 2 - 3 } & Constraints (30) & 288 \\
\cline { 2 - 3 } & Constraints (31) & 288 \\
\cline { 2 - 3 } & Constraints (32) & 288 \\
\cline { 2 - 3 } & Constraints (33) & 144 \\
\cline { 2 - 3 } & Constraints (34) & 288 \\
\cline { 2 - 3 } & Constraint (35) & 1 \\
\cline { 2 - 3 } & Constraints (36) & 36 \\
\cline { 2 - 3 } & Constraints (37) & 144 \\
\cline { 2 - 3 } & Total & 1549 \\
\hline
\end{tabular}

Table B.9: Implementation I performance details

\begin{tabular}{|l|c|c|c|}
\hline Case Study & $\begin{array}{c}\text { Number of runs } \\
\text { converging } \\
\text { successfully }\end{array}$ & $\begin{array}{c}\text { Number of runs } \\
\text { converging } \\
\text { prematurely }\end{array}$ & $\begin{array}{c}\text { Number of runs } \\
\text { crashing due to } \\
\text { integration problems }\end{array}$ \\
\hline Case Study A & 13 & 28 & 9 \\
\hline Case Study B & 22 & 23 & 5 \\
\hline
\end{tabular}



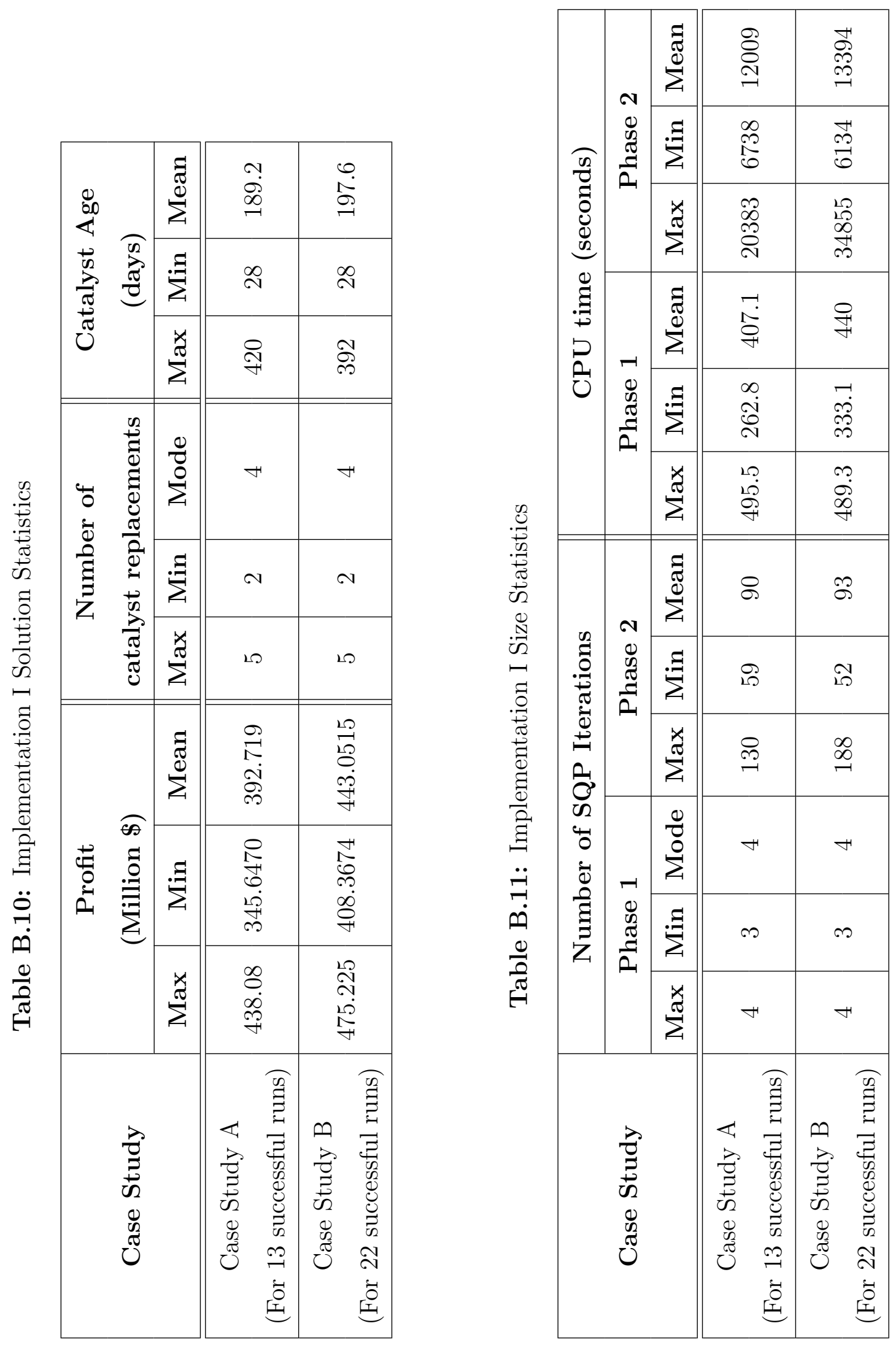

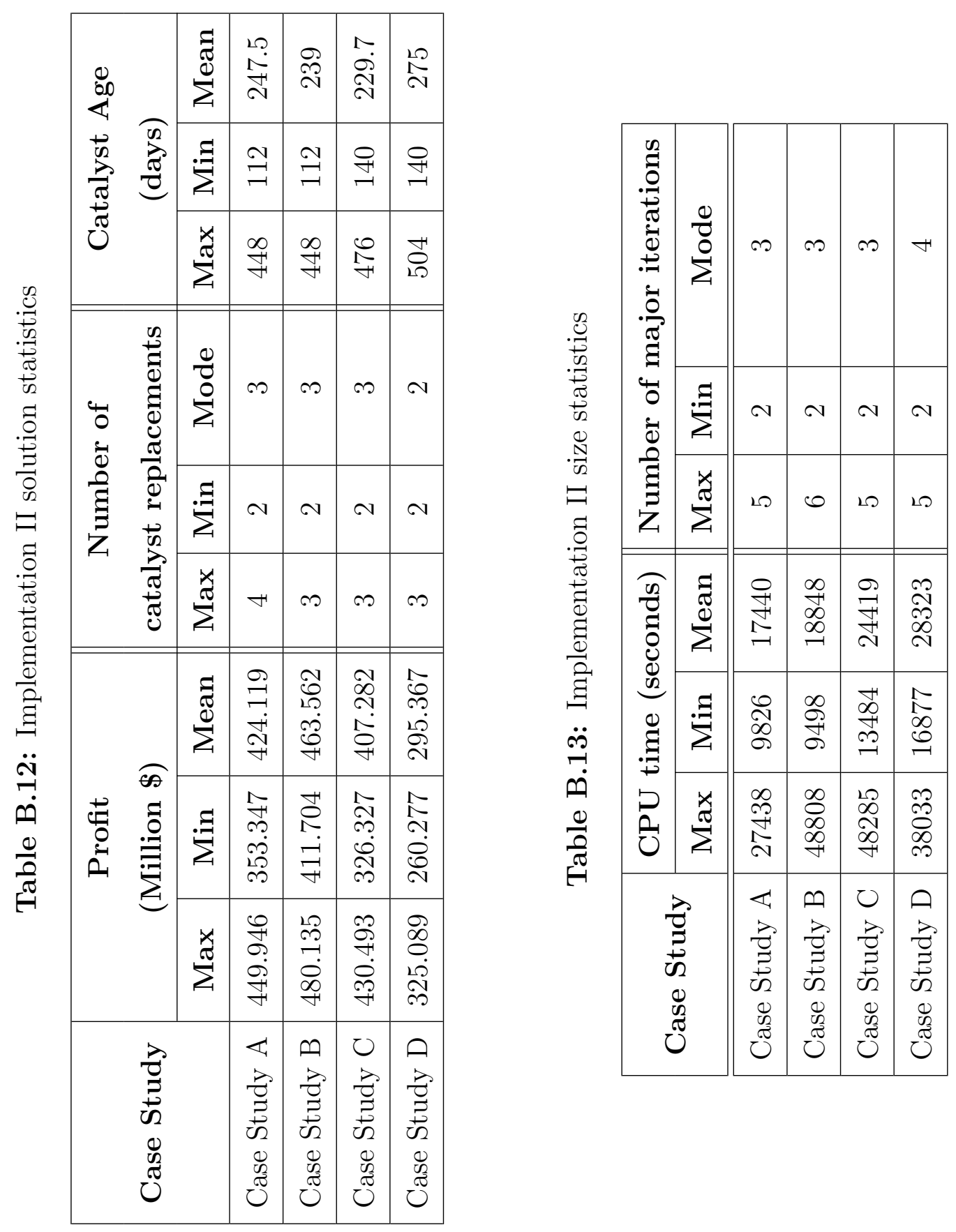


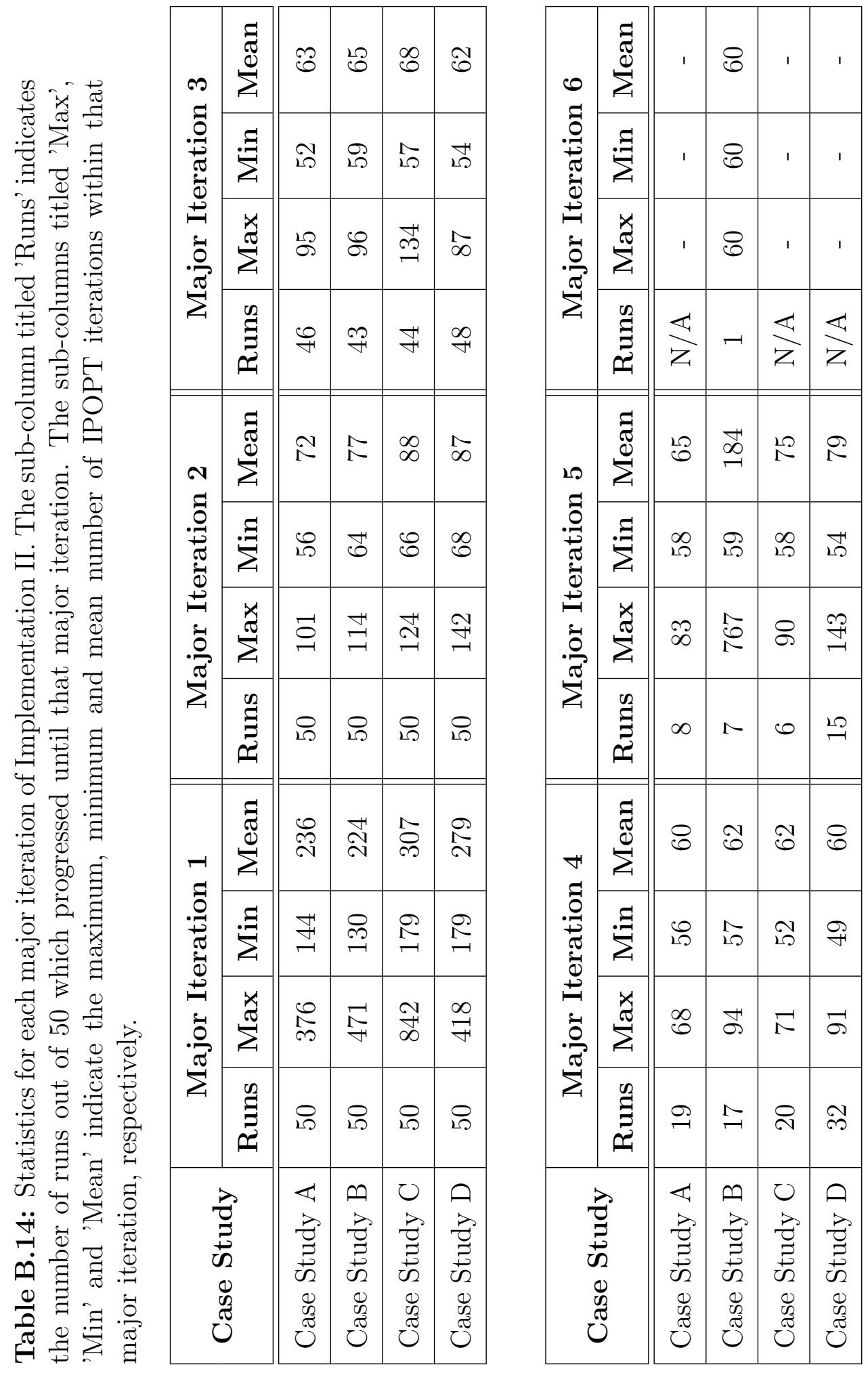




\section{References}

\section{References}

Adloor, S.D., Al Ismaili, R., Wilson, D.I., Vassiliadis, V.S., 2018. Errata: Heat exchanger network cleaning scheduling: From optimal control to mixed-integer decision making. Computers \& Chemical Engineering 115, $243-245$.

Al-Ameri, T.A., Shah, N., Papageorgiou, L.G., 2008. Optimization of vendormanaged inventory systems in a rolling horizon framework. Computers \& Industrial Engineering 54, 1019-1047.

Al Ismaili, R., Lee, M.W., Wilson, D.I., Vassiliadis, V.S., 2018. Heat exchanger network cleaning scheduling: From optimal control to mixedinteger decision making. Computers \& Chemical Engineering 111, 1-15.

Andersson, J., 2013. A General-Purpose Software Framework for Dynamic Optimization. PhD thesis. Arenberg Doctoral School, KU Leuven. Department of Electrical Engineering (ESAT/SCD) and Optimization in Engineering Center, Kasteelpark Arenberg 10, 3001-Heverlee, Belgium.

Belghith, S., Lamnabhi-Lagarrigue, F., Rosset, M.M., 1986. Bang-bang solutions for a class of problems arising in thermal control, in: Algebraic and Geometric Methods in Nonlinear Control Theory. Springer, pp. 623-632.

Bellman, R., Glicksberg, I., Gross, O., 1956. On the bang-bang control problem. Quarterly of Applied Mathematics 14, 11-18.

Bizet, V.M., Grossmann, I.E., Juhasz, N.M., 2005. Optimal production and scheduling of a process with decaying catalyst. AIChE journal 51, 909-921.

Blakemore, N., Aris, R., 1962. Studies in optimization - V : The bang-bang control of a batch reactor. Chemical Engineering Science 17, 591-598. 
Bryson, A., Ho, Y.C., 1975. Applied optimal control: Optimization, estimation, and control (revised edition). Levittown, Pennsylvania: Taylor \& Francis .

Chou, A., Ray, W.H., Aris, R., 1967. Simple control policies for reactors with catalyst decay. Transactions of the Institution of Chemical Engineers and the Chemical Engineer 45, T153.

Crowe, C.M., 1970. Optimization of reactors with catalyst decay I: Single tubular reactor with uniform temperature. The Canadian Journal of Chemical Engineering 48, 576-584.

Crowe, C.M., 1976. Optimization of reactors with catalyst decay and the constant conversion policy. Chemical Engineering Science 31, 959-962.

Crowe, C.M., Lee, S.I., 1971. Optimization of reactors with catalyst decay III: Tubular reactor with several beds of uniform temperature. The Canadian Journal of Chemical Engineering 49, 385-390.

Eronen, V.P., Mäkelä, M.M., Westerlund, T., 2015. Extended cutting plane method for a class of nonsmooth nonconvex MINLP problems. Optimization 64, 641-661.

Geoffrion, A.M., 1972. Generalized Benders Decomposition. Journal of optimization theory and applications 10, 237-260.

Hindmarsh, A.C., Brown, P.N., Grant, K.E., Lee, S.L., Serban, R., Shumaker, D.E., Woodward, C.S., 2005. SUNDIALS: Suite of nonlinear and differential/algebraic equation solvers. ACM Transactions on Mathematical Software (TOMS) 31, 363-396.

Ho, T.C., 1984. Some aspects of the constant-conversion policy dealing with catalyst deactivation. Journal of Catalysis 86, 48-54. 
Houze, M., Juhasz, N., Grossmann, I.E., 2003. Optimization model for production and scheduling of catalyst replacement in a process with decaying performance, in: Proceedings FOCAPO.

Kirches, C., Sager, S., Bock, H.G., Schlöder, J.P., 2010. Time-optimal control of automobile test drives with gear shifts. Optimal Control Applications and Methods 31, 137-153.

Krishnaswamy, S., Kittrell, J., 1979. Analysis of temperature-time data for deactivating catalysts. Industrial \& Engineering Chemistry Process Design and Development 18, 399-403.

Lang, Y.D., Biegler, L., Maier, E., Majewski, R., 2000. An optimal catalyst management strategy for Oxo processes. Computers \& Chemical Engineering 24, 1549-1554.

Ledzewicz, U., Schättler, H., 2002. Optimal bang-bang controls for a twocompartment model in cancer chemotherapy. Journal of Optimization Theory and Applications 114, 609-637.

Lee, S.I., Crowe, C.M., 1970. Optimal temperature policies for batch reactors with decaying catalyst. Chemical Engineering Science 25, 743-744.

Li, Z., Ierapetritou, M.G., 2009. Integrated production planning and scheduling using a decomposition framework. Chemical Engineering Science 64, 3585-3597.

Li, Z., Ierapetritou, M.G., 2010. Rolling horizon based planning and scheduling integration with production capacity consideration. Chemical Engineering Science 65, 5887-5900.

Lin, Y., Du, W., 2018. A Two-Level Optimization Framework for Cyclic Scheduling of Ethylene Cracking Furnace System, in: 2018 IEEE Congress on Evolutionary Computation (CEC), IEEE. pp. 1-8. 
MATLAB, Optimisation Toolbox, 2018. version 9.4.0.813654 (R2018a). The MathWorks Inc.

McKay, M.D., Beckman, R.J., Conover, W.J., 1979. Comparison of three methods for selecting values of input variables in the analysis of output from a computer code. Technometrics 21, 239-245.

Mohler, R.R., 1973. Bilinear control processes: with applications to engineering, ecology and medicine. Academic Press, Inc.

Mouret, S., Grossmann, I.E., Pestiaux, P., 2011. A new Lagrangian decomposition approach applied to the integration of refinery planning and crude-oil scheduling. Computers \& Chemical Engineering 35, 2750-2766.

Nocedal, J., Wright, S., 2006. Numerical optimization. Springer Science \& Business Media.

Pacheco, M.A., Petersen, E.E., 1986. A novel interpretation of temperature versus time curves for deactivating catalyst systems. Journal of Catalysis 98, 380-385.

Pontryagin, L., Boltyanskii, V., Gamkrelidze, R., Mischenko, E., 1962. The mathematical theory of optimal processes, Wiley-Interscience. New York .

Sager, S., 2005. Numerical methods for mixed-integer optimal control problems. Der Andere Verlag Tönning.

Sager, S., 2009. Reformulations and algorithms for the optimization of switching decisions in nonlinear optimal control. Journal of Process Control 19, 1238-1247.

Sager, S., Bock, H.G., Reinelt, G., 2009. Direct methods with maximal lower bound for mixed-integer optimal control problems. Mathematical Programming 118, 109-149. 
Sapre, A., 1997. Catalyst deactivation kinetics from variable space-velocity experiments. Chemical Engineering Science 52, 4615-4623.

Serban, R., 2009. sundialsTB v2. 4.0, a MATLAB Interface to SUNDIALS. Technical Report. Technical Report UCRL-SM-212121, Lawrence Livermore National Laboratory.

Shampine, L.F., Reichelt, M.W., 1997. The MATLAB ODE suite. SIAM journal on scientific computing 18, 1-22.

Shi, H., Chu, Y., You, F., 2015. Novel optimization model and efficient solution method for integrating dynamic optimization with process operations of continuous manufacturing processes. Industrial \& Engineering Chemistry Research 54, 2167-2187.

Su, L., Tang, L., Grossmann, I.E., 2015. Computational strategies for improved MINLP algorithms. Computers \& Chemical Engineering 75, 40-48.

Su, L., Tang, L., Grossmann, I.E., 2016. Scheduling of cracking production process with feedstocks and energy constraints. Computers \& Chemical Engineering 94, 92-103.

Szépe, S., Levenspiel, O., 1968. Optimal temperature policies for reactors subject to catalyst deactivation - Batch reactor. Chemical Engineering Science 23, 881-894.

Tang, B., 1993. Orthogonal array-based Latin hypercubes. Journal of the American statistical association 88, 1392-1397.

Vassiliadis, V.S., 1993. Computational solution of dynamic optimization problems with general differential-algebraic constraints. Ph.D. thesis. University of London, London, England. 
Vassiliadis, V.S., Sargent, R., Pantelides, C., 1994a. Solution of a class of multistage dynamic optimization problems. 1. Problems without path constraints. Industrial \& Engineering Chemistry Research 33, 2111-2122.

Vassiliadis, V.S., Sargent, R.W., Pantelides, C.C., 1994b. Solution of a class of multistage dynamic optimization problems. 2. Problems with path constraints. Industrial \& Engineering Chemistry Research 33, 2123-2133.

Wächter, A., Biegler, L.T., 2006. On the implementation of an interiorpoint filter line-search algorithm for large-scale nonlinear programming. Mathematical programming 106, 25-57.

Wang, Z., Li, Z., Feng, Y., Rong, G., 2016. Integrated short-term scheduling and production planning in an ethylene plant based on Lagrangian decomposition. The Canadian Journal of Chemical Engineering 94, 1723-1739.

Westerlund, T., Eronen, V.P., Mäkelä, M.M., 2018. On solving generalized convex MINLP problems using supporting hyperplane techniques. Journal of Global Optimization 71, 987-1011.

Zandvliet, M., Bosgra, O., Jansen, J., Van den Hof, P., Kraaijevanger, J., 2007. Bang-bang control and singular arcs in reservoir flooding. Journal of Petroleum Science and Engineering 58, 186-200. 